\title{
New Technology for Future Colliders
}

DOE grant \# DE-FG03-95ER40924

2005 Progress Report

from the

Texas Engineering Experiment Station

Science Division
Principal Investigators: Peter McIntyre
Professor of Physics
Al McInturff
(979)845-7727
Senior Scientist
p-mcintyre@physics.tamu.edu
(979)845-6015
mcinturff@phyacc.tamu.edu

This document presents an annual report on our long-term R\&D grant for development of new technology for future colliders. The organizing theme of our development is to develop a compact high-field collider dipole, utilizing wind-and-react $\mathrm{Nb}_{3} \mathrm{Sn}$ coil fabrication, stress management, conductor optimization, bladder preload, and flux plate suppression of magnetization multipoles ${ }^{1}$.

The development trail for this new technology began over four years ago with the successful testing of TAMU1 ${ }^{2}$, a NbTi model in which we put to a first test many of the construction details of the high-field design. We have built TAMU2, a mirror-geometry dipole containing a single coil module of the 3-module set required for the 14 Tesla design. This first $\mathrm{Nb}_{3} \mathrm{Sn}$ model was built using ITER conductor which carries much less current than high-performance conductor but enables us to prove in practice our reaction bake and impregnation strategies with 'free' superconductor. TAMU2 has been shipped to LBNL for testing. Work is beginning on the construction of TAMU3, which will contain two coil modules of the 14 Tesla design. TAMU3 has a design field of 13.5 Tesla, and will enable us to fully evaluate the issues of stress management that will be important to the full design.

With the completion of TAMU2 and the construction of TAMU3 the Texas A\&M group 'comes of age' in the family of superconducting magnet R\&D laboratories. We have completed the phase of developing core technologies and fixtures and entered the phase of building and testing a succession of TAMU3 model dipoles that each build incrementally upon a proven core design. TAMU3 provides a testbed in which we can build a succession of model dipoles in which each new model uses one new winding module coupled with one module from the previous model, and uses all of the same structural elements in successive models. This incremental development should enable us to keep to a minimum the time between the completion and testing of successive models. Each new model will incorporate a particular design element that we wish to evaluate: first the basic TAMU3 structure, then substitute one pancake using highperformance superconductor $\left(3,000 \mathrm{~A} / \mathrm{mm}^{2} @ 12 \mathrm{~T}, 4.2 \mathrm{~K}\right)$, then substitute one pancake using mixed-strand cable, then insert a steel nose to reduce the peak field in the end region of a singlepancake coil. While we are building and testing this succession of TAMU3 models we will develop the tooling and evaluate strategies for flaring the ends of the center double-pancake coil needed for.TAMU4. TAMU4 is a full implementation of the design, culminating in 14 Tesla performance. Pending the proposed increase of budget from the present 3-year-flat budget and 
providing that the tests of each model dipole do not lead to substantial modifications of the design, the time to build and test each succeeding model could be $\sim 9$ months.

Our development effort has taken a long time (nine years so far). It has resulted in a remarkably robust and sophisticated design that effectively manages the most difficult issues that have confounded many previous high-field designs ${ }^{2}$ : eliminating stress concentration ${ }^{3}$, distributing preload uniformly, maintaining uniform preload through cool-down, providing adequate support and cooling for leads and splices, and suppressing snap-back ${ }^{4}$ by an estimated factor of 4 .

A perusal of our proposals and annual reports over the nine years of our program reveals that, while the technology that we set out to develop is in fact maturing, the pace of our development has lagged far behind what we have anticipated in our annual proposals. There are two reasons for this. First, we have at all times approached the awesome challenges of the technical developments with a measure of optimism. Such optimism is an essential ingredient for anyone to actually attack these difficult endeavors. Our optimism has been born out in full in terms of the impact that our program has had in materially advancing the technology of high-field magnets. It has not been born out in the calendar schedule of our developments. The second reason is that, in most years of the program, DOE has funded our renewal at a level substantially below what we requested. This shortfall has disproportionately limited our productivity because of its impact on long-lead critical supplies and fabrication services.

The ultimate objective of our program is to develop TAMU4 as a 14 Tesla field-quality $\mathrm{Nb}_{3} \mathrm{Sn}$ dipole optimized for hadron collider requirements.

We hope to demonstrate that these two designs provide a baseline of viable solutions from which to optimize the design of future hadron colliders.

During the present funding year we made a sequence of innovations that have major potential benefit for the commissioning of LHC, upgrade of its luminosity, and its long-term future:

- An electrode assembly, suitable for integration within the existing LHC dipoles, capable of killing the electron cloud effect - an effect that threatens to limit the luminosity that could be attained in LHC;

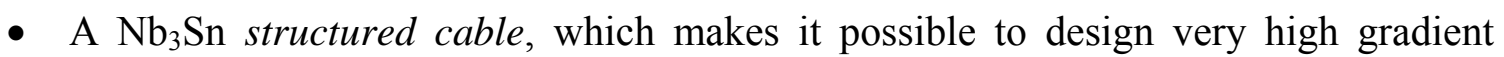
quadrupoles for upgrade of the interaction regions of LHC to enhance its luminosity;

- $\quad \mathrm{A} \mathrm{Nb}_{3} \mathrm{Sn} / \mathrm{NbTi}$ levitated-pole dipole for use in the D1 bends that combine and separate the beams at the intersection regions. The levitated-pole design uniquely solves the problems of radiation damage and heating from particles swept from the beam.

- A hybrid dipole technology, in which inner windings of Bi-2212 are integrated in a $\mathrm{Nb}_{3} \mathrm{Sn}$ block-coil dipole to push to 24 Tesla, opening the possibility of a future tripler upgrade of $\mathrm{LHC}^{5}$.

We believe that these contributions place us in the leadership of the effort to maximize the performance of LHC for the world high energy physics program. 


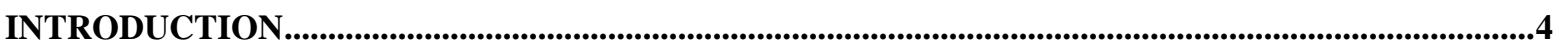

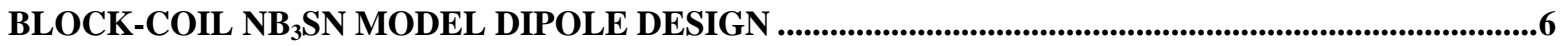

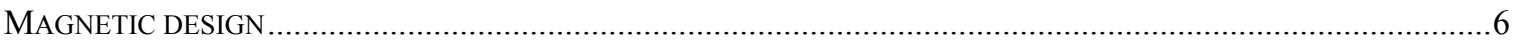

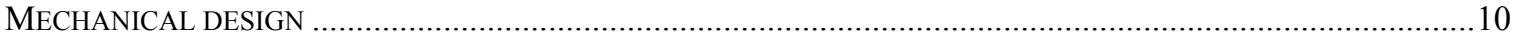

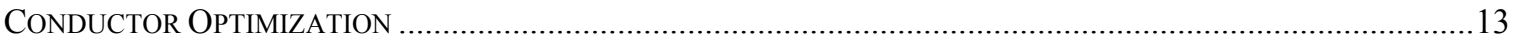

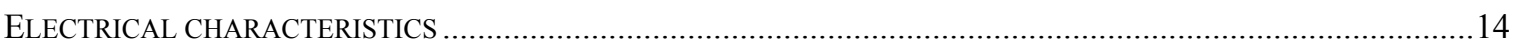

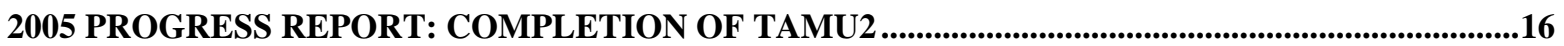

PROVISIONS FOR MODULE SUPPORT AND GAS/EPOXY FLOW MANIFOLDS IN THE THICK SKIN ……...................16

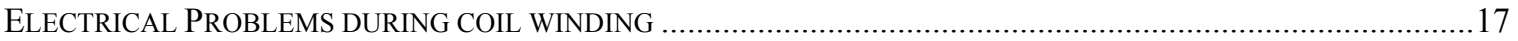

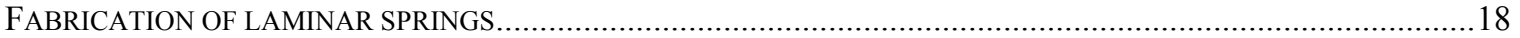

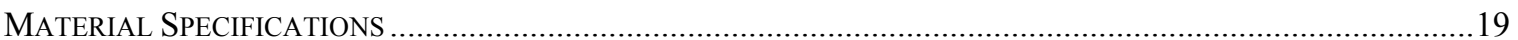

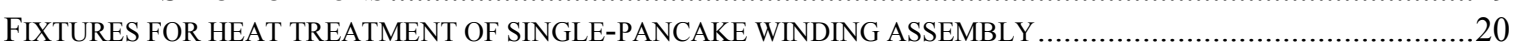

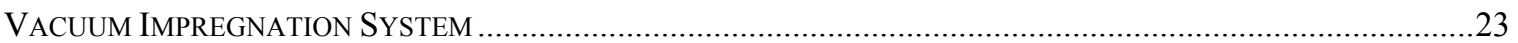

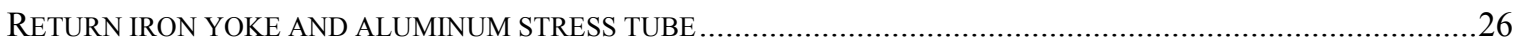

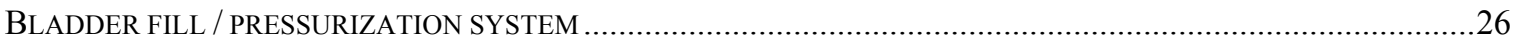

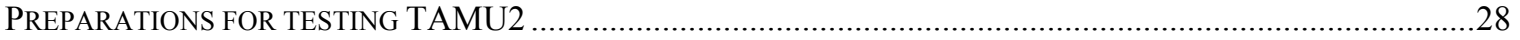

2005/2006 WORK PLAN............................................................ERROR! BOOKMARK NOT DEFINED.

CONTRIBUTIONS OF THE TAMU PROGRAM TO LHC COMMISSIONING AND UPGRADE.....30

ELECTRODE ASSEMBLY TO KILL ELECTRON CLOUD EFFECT IN THE LHC ARC DIPOLES ..................................31

STRUCTURED CABLE QUADRUPOLES FOR Q1 QUADRUPOLES IN LHC LUMINOSITY UPGRADE ..........................32

LEVITATED-POLE DIPOLE FOR DI DIPOLE IN LHC LUMINOSITY UPGRADE ....................................................3

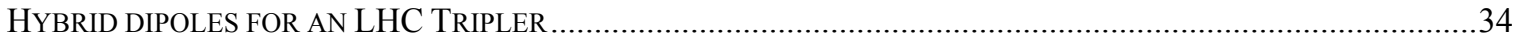

BUDGET REQUEST ................................................................ERROR! BOOKMARK NOT DEFINED.

CONTRIBUTED EFFORT BY TEES AND TAMU ……...................................ERROR! BOOKMARK NOT DEFINED. SUPPORT REQUIRED OUTSIDE OF THE REQUESTED DOE GRANT FUNDING .ERROR! BOOKMARK NOT DEFINED.

KEY PERSONNEL............................................................................................................................................35

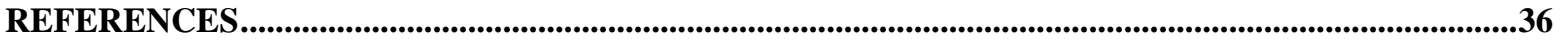




\section{Introduction}

The accelerator research group at Texas A\&M University is developing dipole technology for use in future hadron colliders. The goal of our research is to extend superconducting dipole technology in three respects that are key to its role in extending the capabilities for future hadron colliders:

- Manage the stress build-up and tolerance issues that are primary limitations to cost and reliability in high-field dipoles;

- Reduce the multipole fields that are produced during snap-back so that the filament size of $\mathrm{Nb}_{3} \mathrm{Sn}$ superconducting wire does not limit dipole performance in collider dipoles $^{4}$;

- Improve the magnetic efficiency (central field strength/area of superconducting coil) so that less expensive superconductor is required for the dipoles of a collider.

We suggest that these issues will pace the viability of proposals for major new facilities for both hadron colliders and muon colliders, two of the three cornerstones of the future of high energy physics.

The Texas A\&M group has pioneered several innovations in magnet design ${ }^{1}$ with the objective of achieving 14 Tesla in a cost-effective, manufacturable dipole: the use of rectangular pancake coils, stress management and shear release within the coil assembly, suppression of persistent current multipoles; and mixed-strand cables that incorporate pure copper strands for quench protection. While each of these elements has been used in one form or another in the past for other applications, we have combined them to particular advantage to control stress within the fragile $\mathrm{Nb}_{3} \mathrm{Sn}$ coils of a high-field dipole ${ }^{3}$ and to dramatically reduce the amount of superconductor actually needed to achieve a given field strength ${ }^{6}$.

Four years ago we successfully tested TAMU1, a first NbTi learning model of the block-coil technology. It reached its short-sample limit without training. This is the more remarkable because it one of the few high-field NbTi dipoles ever built with vacuum-impregnated coils (LBNL's D-10 was another such). It has long been thought that NbTi dipoles must be built with B-stage insulation in which the coil is bathed in liquid helium during operation for stability. The enthalpy of the small amount of helium in the interstices of the coil provides substantial stabilization against microquenches in the event that a region of conductor moves microscopically during excitation. We interpret TAMU1's success as a validation that coil motion is sufficiently well controlled in the block-coil design that this extra stabilization is not needed. This result is crucial for reliable operation of $\mathrm{Nb}_{3} \mathrm{Sn}$ coils, because they must be vacuum impregnated.

This spring we completed TAMU2, a first $\mathrm{Nb}_{3} \mathrm{Sn}$ model of our dipole technology. This learning model is built using a cable composed of ITER strand, and will produce 5.2 Tesla at the center probe and 7.6 Tesla maximum in the winding. In its design we utilize all of the features of pancake coil geometry and stress management for our high-field $\mathrm{Nb}_{3} \mathrm{Sn}$ design, and we also adopt two innovations that the LBNL group has made in their development of superconducting dipoles: self-contained pancake coil subassemblies ${ }^{7}$ that eliminate the need for external control of axial loading, and expandable stainless steel bladders to deliver uniform preload to the coil support structure and relax dimensional tolerances ${ }^{8}$. 
The TAMU2 design also incorporates a new innovation that we devised two years ago. A thin steel sheet is located between the center double-pancake winding and the flanking singlepancake windings. This horizontal flux plate presents a dipole boundary condition that is closecoupled to the beam tube region. At injection field the steel is unsaturated and provides a strong suppression of persistent-current multipoles and snap-back. The suppression roughly compensates for the much larger filament sizes in currently available $\mathrm{Nb}_{3} \mathrm{Sn}$ compared to $\mathrm{NbTi}$. This flux plate design can only be implemented in the block coil design (coil placement in $\cos \theta$ and common-coil geometries cannot accommodate it).

TAMU2 contains a single two-winding pancake coil module of ITER cable, configured in a mirror-geometry flux return. The second model (TAMU3) contains two modules, each with the same structure as the one used in TAMU2, but using high-performance $\left(2500 \mathrm{~A} / \mathrm{mm}^{2}\right)$ internalSn conductor.

We have completed TAMU2 and shipped it to LBNL for testing. We plan to test in August 2005 , pending budget arrangements from DOE to LBNL to fund the tests.

We have begun the fabrication of piece-parts for TAMU3. We have made an immense investment in the development of tooling and fixtures for winding, reaction bake and vacuum impregnation of these model magnets. The succession of TAMU3 dipoles will all use the same tooling and fixtures, so their construction and testing should proceed in a more predictable and expeditious schedule.

We participated in the 2003 Workshop 'Super Magnets for Super Colliders' at Erice. At that workshop a set of priorities for future collider magnet R\&D were epitomized. These included as a milestone a 14 Tesla model dipole with collider-quality fields. TAMU4 should accomplish that milestone and is the goal of our program over the next several years.

Stimulated by the new focus provided at that workshop, we also began to ask how Bi-2212 windings could be incorporated into a structured dipole to push field strength even higher than the limits imposed by $\mathrm{Nb}_{3} \mathrm{Sn}$. Those questions brought an answer in Fall 2004 with our invention of the hybrid dipole TAMU5 ${ }^{5}$, incorporating inner windings of Bi-2212 where the field in coil is highest, to produce a design that should be capable of 24 Tesla. If that dipole could be made practical, it would provide a basis for a future tripling of the energy of LHC as an upgrade in the same tunnel.

Finally we have applied the techniques of stress management in a very different accelerator magnet application, to design a flux-coupled stack of isochronous cyclotrons that could produce 7 independent $800 \mathrm{MeV} 2 \mathrm{~mA}$ proton beams to drive a GW thorium-cycle fission power reactor ${ }^{9}$. One element of that design has now received seed funding. If the development proves to be practical for large-scale power generation, it could be the most important industrial application of particle accelerators ever made.

This renewal proposal seeks funding for the continuation of our research in 2006. During that year we plan to complete and test a first model of TAMU3, and to start construction of a second model designed to test the same coil construction with the best state-of-the-art superconductor available. In the subsequent year we will complete and test the second TAMU3 and build a model dipole that incorporates a central doublet winding to complete the geometry of TAMU4. We stress that the pace of our program towards these goals will be strongly limited or boosted according to the decision by DOE to fund the program at the proposed level. 


\section{Block-coil $\mathrm{Nb}_{3} \mathrm{Sn}$ model dipole design}

We will first describe the design of the block-coil $\mathrm{Nb}_{3} \mathrm{Sn}$ dipole that forms the basis for our current series of model dipoles. Figure 1 shows an isometric view of the coil assembly with one quadrant of the flux return steel.

\section{Magnetic design}

We have made an important improvement in the dynamic range and suppression of persistent-current multipoles by introducing a steel sheet between the center double-pancake winding and each outer pancake winding, as shown in Figure 2. The calculated field distribution at injection field (0.6 Tesla) is shown in Figure 5. At low field strength, the steel sheet presents an unsaturated planar boundary condition that is close-coupled to the beam aperture. This boundary condition is effective in suppressing both geometric multipoles and multipoles arising from persistent-current magnetization in the superconducting elements (shown in Figure 3 for the highperformance strand to be used in our outer windings). In particular, this suppression is linear and therefore equally effective in suppressing the flux change associated with "snap-back", as contrasted to schemes involving the placement of saturated magnetic elements around the beam tube.

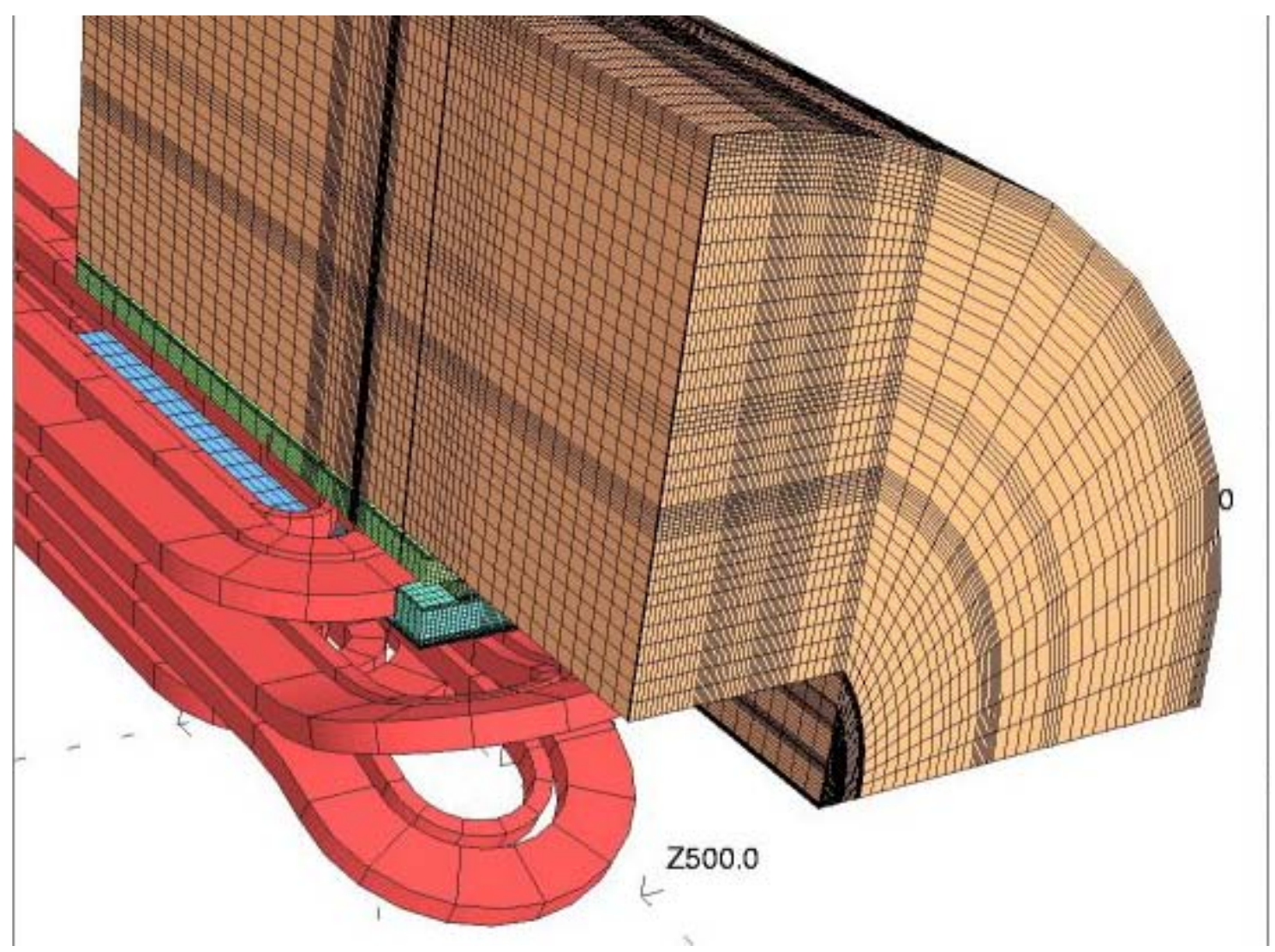

Figure 1. Isometric view of the coils in the block-coil $\mathrm{Nb}_{3} \mathrm{Sn}$ dipole, with one quadrant of the flux return steel shown. 


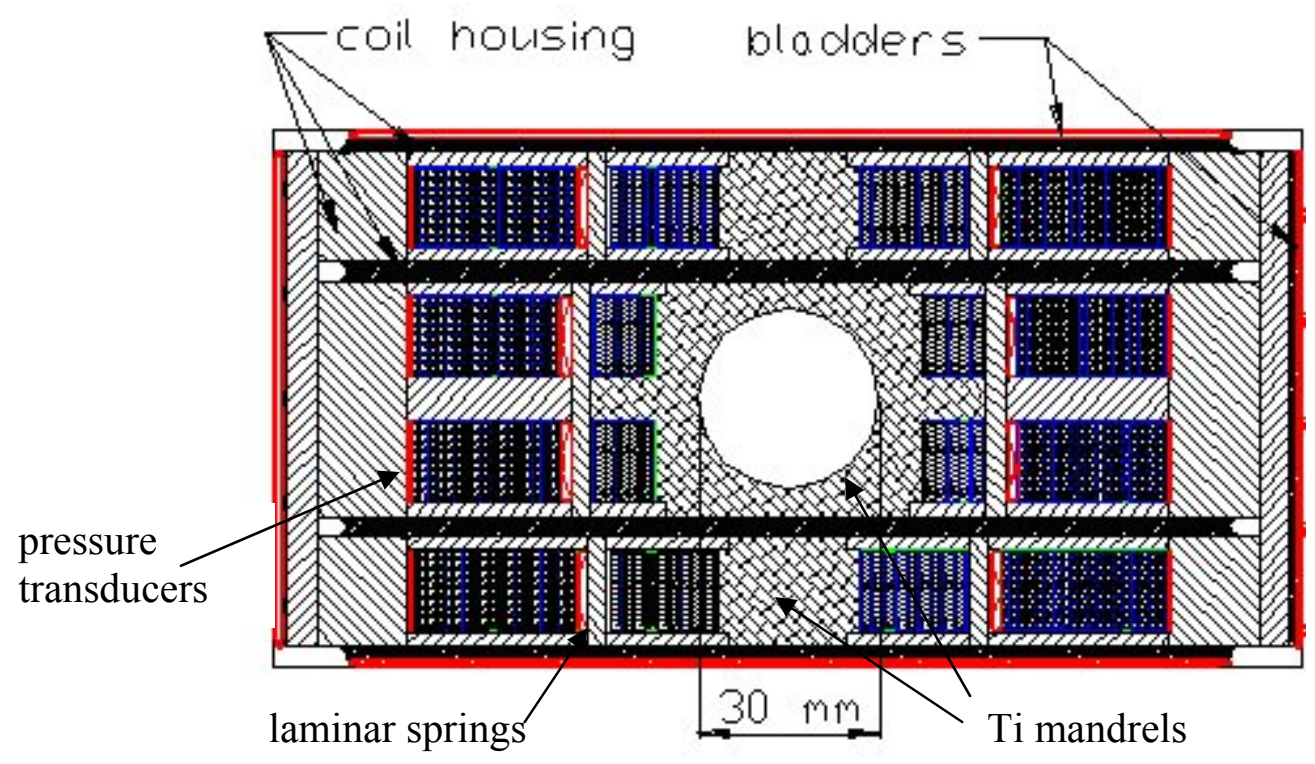

Figure 2. Detail of coil structure and placement of bladders and flux plates.

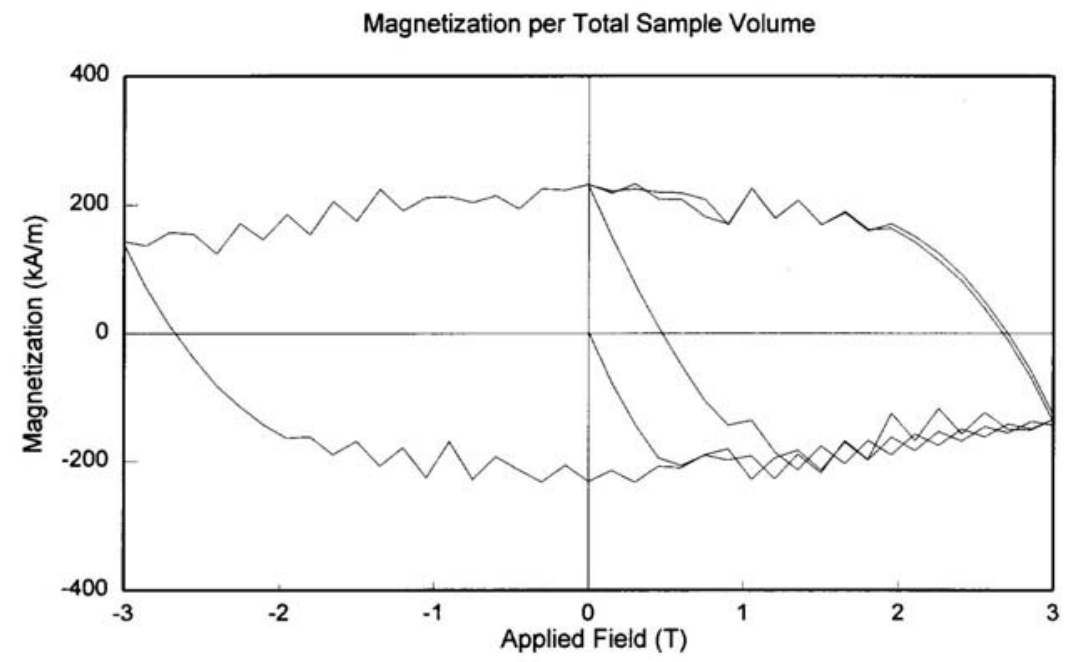

Figure 3. Measured magnetization curve of OSC superconducting strand. ${ }^{10}$

All windings can be powered in series from a single supply over the entire dynamic range. Figure 4 shows the calculated multipoles over a 20:1 dynamic range. All terms stay less than a few units $\left(10^{-4} \mathrm{~cm}^{-\mathrm{n}}\right)$ even at the worst field, $6 \mathrm{~T}$, where steel saturation is progressing deeper into the flux return.

The filament diameter in currently available $\mathrm{Nb}_{3} \mathrm{Sn}$ strand is $30-80 \mu \mathrm{m}$, ten times that in $\mathrm{NbTi}$ strand. The resulting impact on persistent-current magnetization is one of the greatest challenges facing the development of high-field collider dipoles ${ }^{4}$. At the Tevatron and LHC, persistent-current multipoles are one of the most serious concerns affecting injection and emittance growth during acceleration. The worst aspect of persistent current multipoles is the snapback phenomenon, when the magnetization snaps from the semi-dc hysteresis curve to the ramping value as the current is increased by a few amperes from its injection or accumulation flat-top as acceleration begins. 


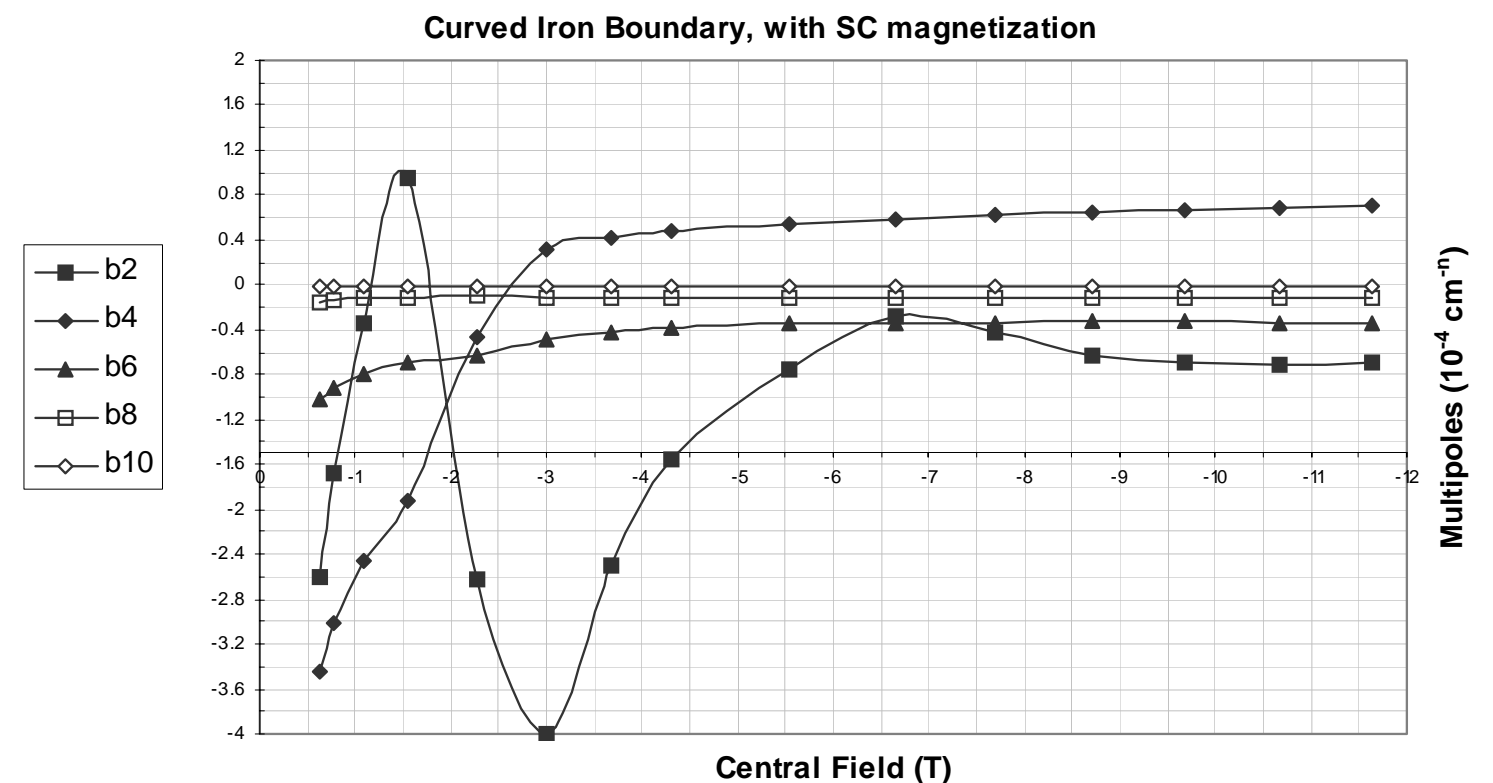

Figure 4. Calculated multipoles including persistent current magnetization.

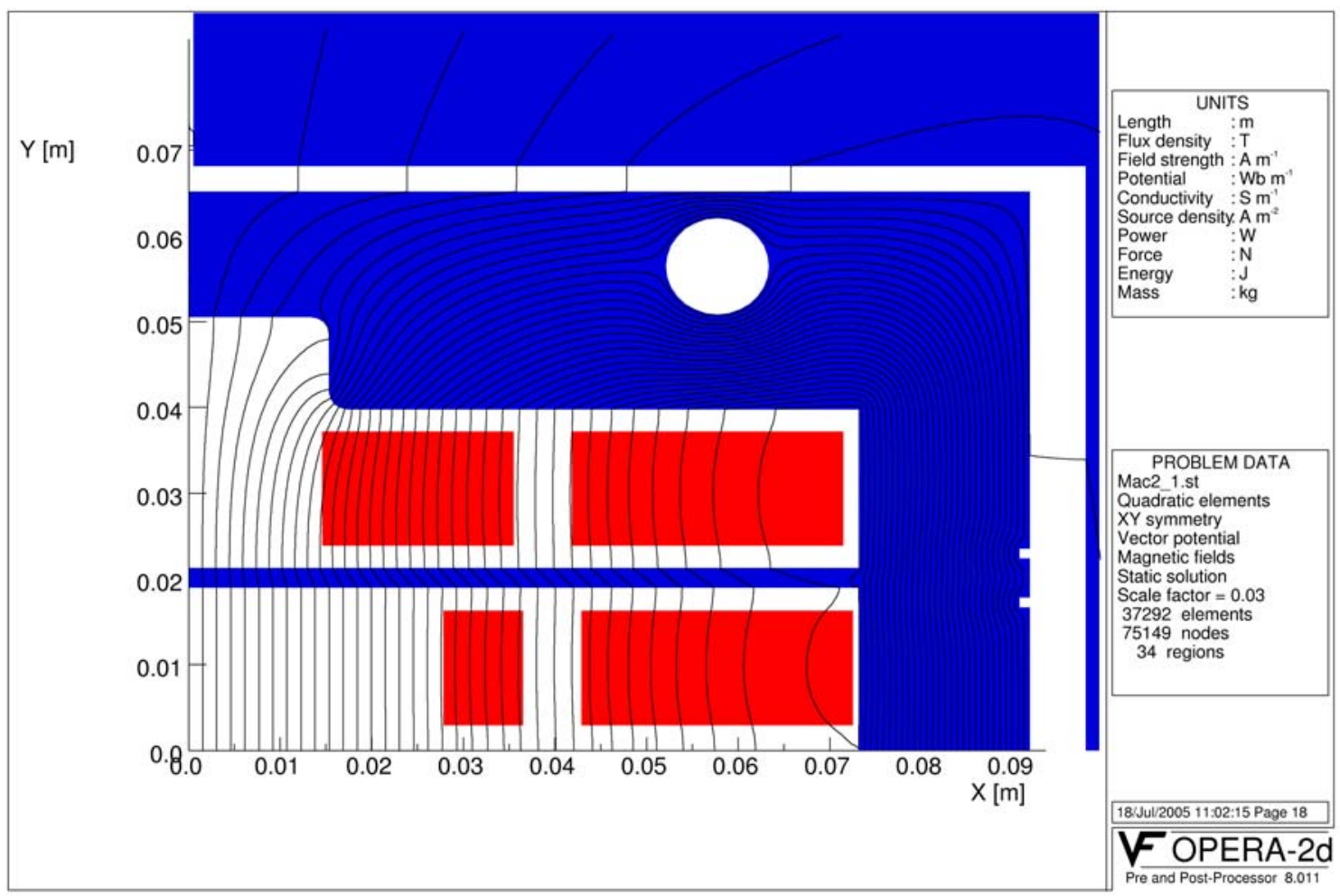

Figure 5. Calculated body field distribution in TAMU4 dipole $(0.5 \mathrm{~T})$. 
Several schemes to correct persistent-current fields have been suggested, including passive superconducting beam-tube windings, and distributions of ferromagnetic material. They have the common problem that the multipoles from $\mathrm{Nb} 3 \mathrm{Sn}$ strand are at least ten times greater than that for the NbTi dipoles of current colliders, where the problems are already serious.

The block-coil dipole uniquely delivers a natural fourfold suppression of persistent-current sextupole, as a benefit from its close-coupled planar steel boundary over and under the beam tube region ${ }^{11}$. At low field these boundaries are unsaturated, and strongly suppress multipoles. This suppression is clearly evident in Figure 5, which shows the lines of force at a dipole field strength of 0.5 Tesla (injection field). The lines of force re-distribute in the unsaturated steel flux plate (the blue sheet above the beam tube), yielding pure dipole field in the beam tube region. We have calculated the persistent-current multipoles in the block-coil design, using the effectivemagnetization technique of Caspi, assuming a filament size of $50 \mu \mathrm{m}$. The persistent-current sextupole was nearly identical to that measured in the SSC dipoles. The block-coil geometry is the only high-field design for which the persistent-current magnetization from currently available $\mathrm{Nb}_{3} \mathrm{Sn}$ conductor is nearly acceptable for collider requirements.

It is interesting to note that we plan to construct two models of TAMU4 (when we get that far!), one with the iron flux plate and one with a stainless steel substitute. We can use the same winding modules, simply dis-assembling the first, and substituting the alternate flux plate, and re-assembling and re-pressurizing the bladder preload. Comparison of persistent magnetization multipoles and snapback in the two cases will give the first direct measurement of a technique to suppress snapback, and could provide a test basis for future work on the subject.

End field control. We re-designed the end region of the coils to eliminate the maximumfield point that would occur at the inside of the coil bends. We spaced the bends in the inner and outer windings as shown in Figure 1, and inserted stainless steel end laminations in the flux return. We were able thereby to reduce the end fields so that they do not limit magnet performance. The re-designed ends and the field distribution are shown in Figure 6.

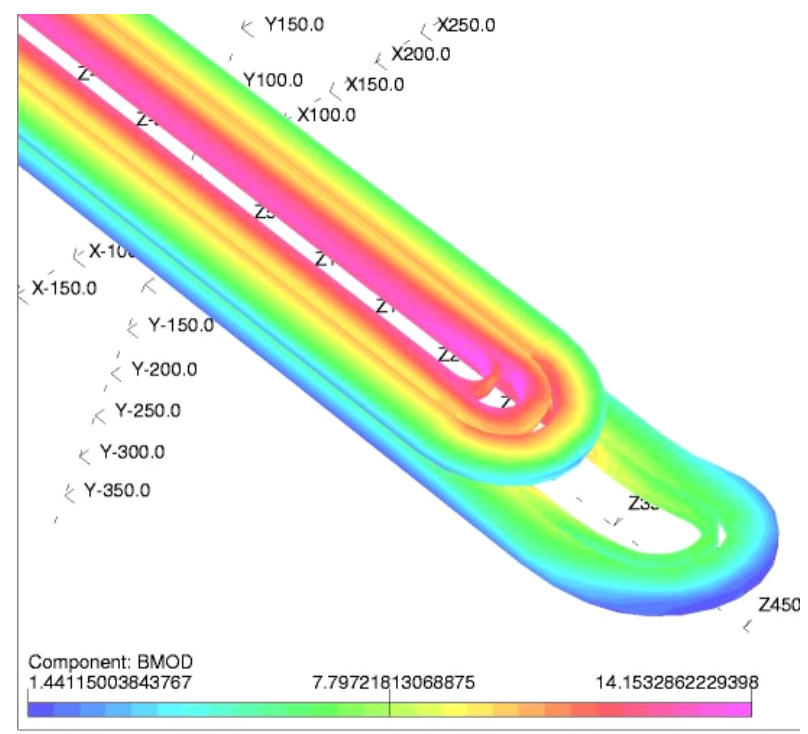

Figure 6. Distribution of field strength in the windings, showing elimination of peak field in ends. (The iron yoke is suppressed in the figure for clarity). 


\section{Mechanical design}

Stress management. The block-coil dipole shown in Figure 2 incorporates the provisions for stress management that we pioneered in our earlier block-coil designs ${ }^{3}$. It also incorporates two innovations that the LBNL group has introduced into their common-coil dipole. First, the coil is divided into three subassemblies - a central double-pancake and two flanking single pancakes. Each subassembly is separately sealed within a housing and vacuum impregnated. This approach enables us to support the axial loads on each of the top and bottom coils within its housing, eliminating the need for any further provisions in the overall structure. The ends of each winding in the center double-pancake are spread to accommodate the beam tube. Since its ends extend beyond the ends of the flanking windings and beyond the flux return, the axial forces are modest and easily controlled by end blocks.

Winding structure. Each of the outer windings is packaged as an independent unit, as shown in Figure 7. Each assembly consists of a 'thick skin' backing plate, made of magnetic steel, with the Ti winding mandrel keyed into a channel in the thick skin. The leads are brought out in channels in the thick skin. Once winding is complete the coil remains attached to its mandrel and thick skin through the balance of fabrication, heat treatment, impregnation, and assembly.

Once winding and reaction bake are complete, a structural package is completed around the coil. The ground plain insulating sheet (containing quench heaters and voltage taps) is applied to the exposed surface of the windings, then the steel flux plate is applied to cover the ground plane, then side rails were positioned before the heat treatment. The assembly is already compressed in both transverse and longitudinal dimensions and the seams of the side rails and skins are welded to lock the coil under pre-compression of the spring and contraction of the coil. The leads emerge at the ends of the package, and are each spliced to double-cable NbTi leads. The splice joint is completely supported within an extension of the thick skin, providing rigid support to the critical pre-lead cable region. The module is then vacuum-impregnated with CTD101 epoxy.

Bladder preload. Second, the coil structure is preloaded within the flux return by an arrangement of expandable bladders (a vertical and a horizontal circuit), and the flux return is preloaded within a super-alloy aluminum shell in the same manner. The bladders are filled with a low-melt-temperature metal alloy. The alloy is pressurized while molten, and the assembly is cooled to solidify it while maintaining the preload pressure. In this way we dramatically relax tolerances on all outer elements, deliver independent preloads to the coil structure and the flux return, and provide for additional preload to be delivered by differential contraction during cooldown to liquid helium temperature.

Figure 7 illustrates the geometry for the bending of the center windings at the ends to accommodate the beam tube. Figure 2 shows the arrangement of the inner and outer bladders on the final cross section. Figure 7 illustrates another important feature of the TAMU2 design. Transverse preload and support are provided by pressurizing bladders filled with Wood's metal. Clyde Taylor used this technique successfully for a superconducting sextupole magnet. The bladders are located on all four faces of the coil assembly, and in the annular space between the flux return steel and an outer aluminum shell. The aluminum shell is the structural element that provides overall containment of all forces.

To complete the dipole, the flux return halves are inserted into the aluminum shell with the vertical split bladders and outer bladders collapsed. The magnet assembly is heated above the 
melt temperature of the Wood's metal filling, the outer bladders are pressurized, and the assembly is cooled to freeze in the compression. The magnet assembly is then returned to a horizontal orientation and the inner bladders (collapsed) are inserted into the inner rectangular slots flanking the coil assembly. The entire assembly is re-heated above the melt temperature of the filler metal, and the inner bladders are evacuated and back-filled with molten filler metal. The molten metal is pressurized to deliver preload to the coil structure. The dipole is then cooled to room temperature, solidifying the alloy while maintaining the pressure inside the bladders. When the dipole is subsequently cooled to liquid helium temperature, the differential shrinkage of the aluminum delivers additional preload to the flux return therefore keeping the polar shield gap closed.

This approach has the advantage that uniform preload is delivered everywhere, independent of variations in the gap between elements due to tolerances. It has the further advantage of simplicity in the otherwise complex task of delivering and monitoring preload.

Stabilization of dimensions during cooldown using Ti elements. The differential contraction of the superconducting coil and the Inconel structural elements opens the risk of losing some compression of the coil during cooldown. To avoid this, and because the innermost winding is not spring-loaded, we fabricated the mandrel elements blocks of Ti within the structure, as shown in Figure 2. Since Ti shrinks less than any of the other constituent metals, the preload is preserved during cooldown.

Pressure transducers. We install pressure transducers at the outer boundaries of the outer coils (see Figure 1) so that we can measure the total force delivered on the outer winding and verify that stress management is operating as planned.

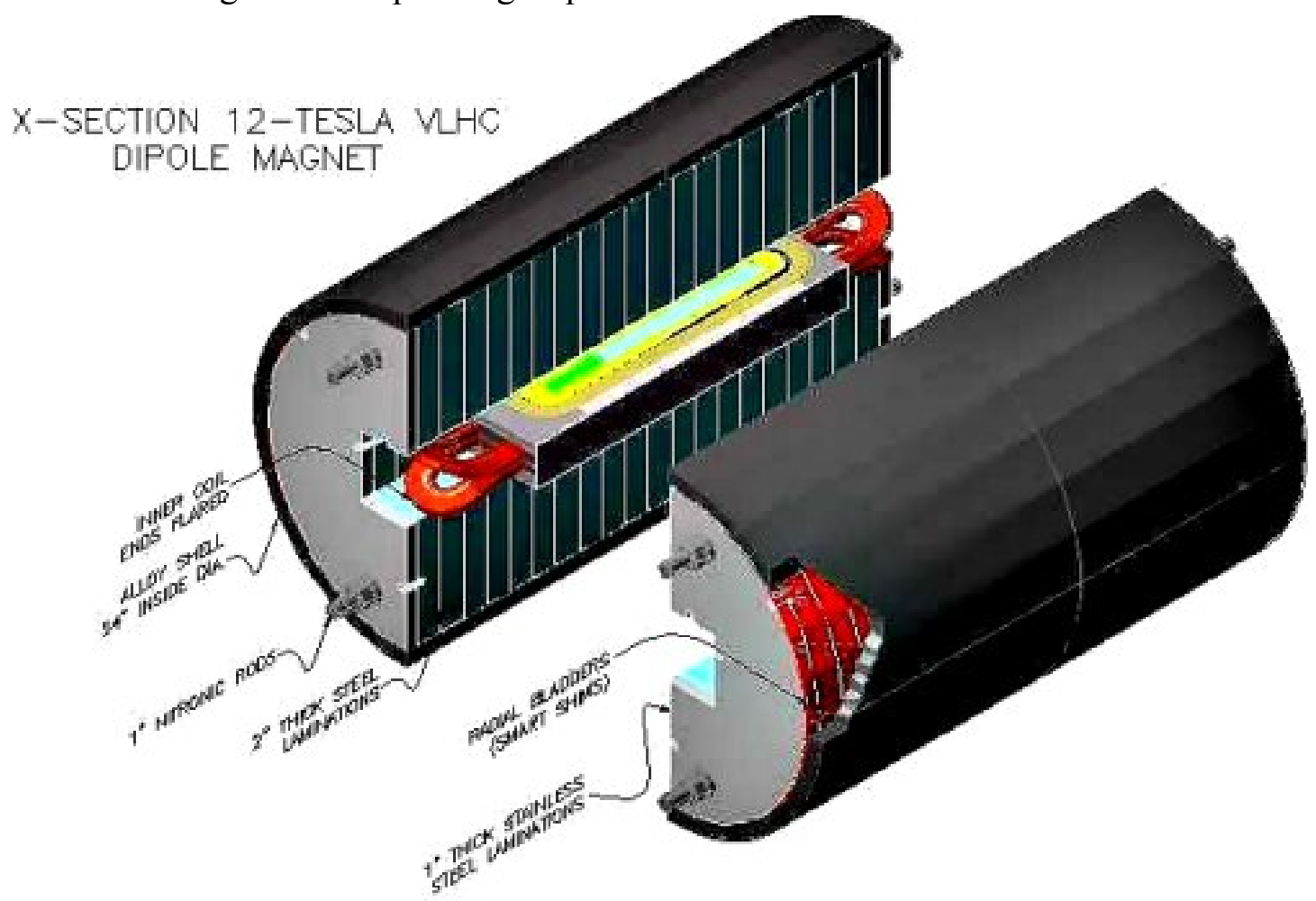

Figure 7. Blow-up assembly of the 12 Tesla dipole - center ends bent $\pm 25^{\circ}$. 

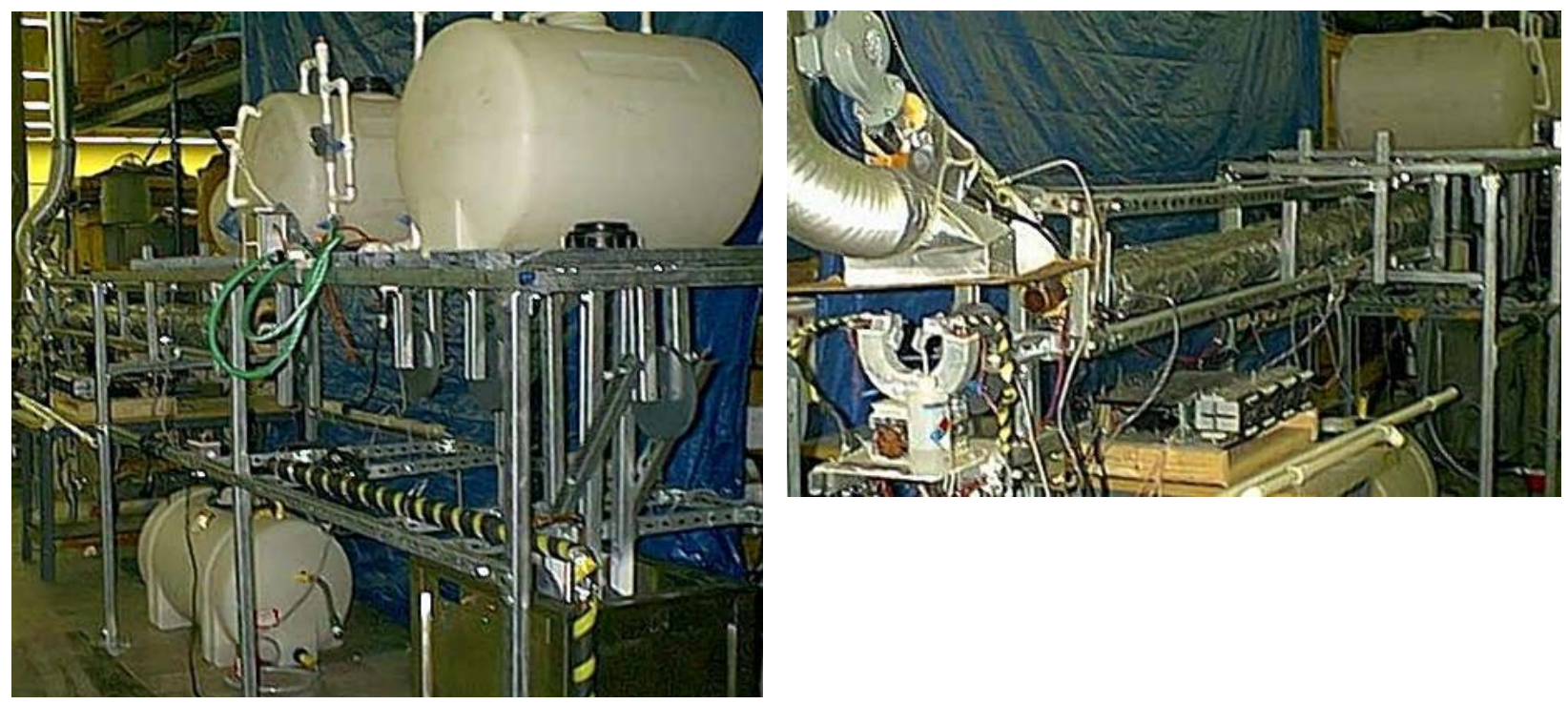

Figure 8. (a) Detergent wash, rinse stations; (b) palmitic acid atomizer and dryer process.

Pre-anneal. The cable is taken up onto a metal spool and subjected to a copper relaxation anneal (160 C, $2 \mathrm{hr}$ ) and then re-sized. Dietderich at LBNL has found that this pre-anneal serves to relax much of the deviatoric stress from strand manufacture, so that there is less net contraction during the A-15 formation heat treatment cycle ${ }^{12}$.

S-glass insulation: weaving, cleaning, re-sizing. S-glass fabric insulation is woven directly onto the cable on a process line at New England Electric Co. The direct-weave approach gives better uniformity of coverage, therefore a thinner and more efficient insulator than does a pulledon sock. It does however require that the S-glass be woven on with starch sizing applied to the $\mathrm{S}$-glass filaments to lubricate the weaving process. We must remove the sizing before winding the coil so that there is no starch to decompose during reaction bake and produce semiconducting carbon in the coil package.

The starch sizing on the cable Fiberglas insulation is removed by passing the cable through an ultrasonic tank containing heated detergent solution (Alconox), then through a second ultrasonic tank containing heated de-ionized water to remove the detergent, as shown in Figure 8a. After cleaning the S-glass we apply a spray mist of a solution of palmitic acid to the two side edges of the insulated cable (Figure $8 \mathrm{~b}$ ). The palmitic acid provides lubrication where wear occurs (where the cable rubs on the coil mandrel as we bend it around at the ends of the coil), but not along the flat sides where there is minimal potential for wear. Palmitic acid volatizes without ash residue during the ramp-up of reaction bake with an intermediate step at $340^{\circ} \mathrm{C}$.

Quench heaters. The block-coil packages are modular, with each unit self-contained in the stress management support structure. The outer winding is loaded against the internal springs next to the support structure rib (Ti). The winding package support plate and rib surfaces are designed to facilitate a cover gas and subsequent epoxy flow pattern into and through the windings, so that we can maintain a purge flow of inert gas during reaction bake and later assure full epoxy penetration during vacuum impregnation.

The quench heater geometry has been redesigned since the NbTi model test. One cover plate on each winding module is removable and after the reaction heat treatment it is removed. 
The voltage taps and spot heaters are attached to their respective traces on the composite Kapton $/ \mathrm{SS} / \mathrm{Cu} / \mathrm{Kapton}$ photo etched circuit, which contains the protection heater elements as well. A layer of Fiberglas and mica paper is placed on top of it and the plate is replaced. The unit is already compressed at size and held by a fixture. This pre-compression of the coil module removes the soft-modulus region of compression so that the windings are in their approximate final cross-section within the support structure prior to heat treat and vacuum impregnation.

\section{Conductor Optimization}

It has long been the practice in superconducting dipole design to grade the conductor. In the outer regions of the coil where the magnetic field is less, and the $\mathrm{j}_{\mathrm{c}}$ in the superconductor is correspondingly larger, a larger fraction of copper stabilizer is incorporated into the strand so that the current density in the copper stabilizer during quench does not exceed safe values for the MIITS and therefore limiting the maximum temperature in the coil after quench ${ }^{13}$. In NbTi the cost of this incorporation of the stabilizing copper was modest and therefore to do so was efficient. In $\mathrm{Nb}_{3} \mathrm{Sn}$ at present, however, the $\sim \$ 800 / \mathrm{kg}$ cost is almost independent of copper content and stabilizing copper using present fabrication techniques becomes a significant cost item within the entire dipole.

We introduced the concept of separating much of the stabilizing copper into pure $\mathrm{Cu}$ strands, cabled with superconducting strands to achieve the desired quench protection. The LBNL group has been trying to develop hybrid cables following our design ${ }^{7}$. They have had some success, but the cabling is difficult because $\mathrm{Cu}$ is considerable softer in its drawing properties than $\mathrm{Nb}_{3} \mathrm{Sn}$ strand even when the $\mathrm{Cu}$ is full hard. They are also exploring an alternative approach in which the stabilizing copper is included as a strip within the cable core.

The conductor optimization strategy pays off particularly well in a block-coil geometry, where the grading of field within the coil is very uniform and permits optimum conductor reduction. The separated-copper approach reduces the total amount of superconducting strand in a dipole by about $40 \%$ in our block-coil designs. Indeed the block-coil dipole with conductor optimization requires significantly less superconductor than any competing design done so far.

The LBNL group has spent considerable effort over the past 2 years in developing mixedstrand cable and in building and testing sub-scale coils using it. Because the copper strands are softer than the superconductor strands (even using full-hard copper) the locking of the Rutherford geometry during cabling is not as effective as in a cable using all superconductor strands. In the prototype coils that have been fabricated with mixed-strand cable, severe training is observed.

We believe that this training may be due to uneven compression and the lack of sustained loading stress during reaction bake in the LBNL winding design. If that conjecture were correct, our laminar springs may give sufficient compressive stress throughout reaction bake to prevent relaxation of an imperfectly locked cable segment. To test this hypothesis, we plan to build one additional single-layer subassembly using mixed-strand cable from LBNL. After testing of the TAMU3 double-pancake assembly, we plan to substitute this mixed-strand subassembly for one of the all-superconductor subassemblies and test that hybrid assembly. That test should give us a first measure of whether the TAMU approach to coil structure provides enough stress control to forgive imperfections in cable locking. It will also be of interest to see if the pure-copper strands anneal during the pre-anneal and re-sizing of the cable. 


\section{Electrical characteristics}

Table 1 presents the vital statistics on the coils for each of a succession of three embodiments of the dipole: one single-pancake coil (TAMU2), two single module high performance cable modules dipole (TAMU3), and the center double-winding plus the two single outer modules (TAMU4), in which the outer windings use cable with half superconducting strands and half pure copper strands in a second remake if funds are available.

Figure 9 presents the load lines for three model dipoles: TAMU2, containing a singlepancake subassembly using ITER conductor; TAMU3, containing two single-pancake subassemblies using high-performance superconductor (3 kA/mm² @ 12 T, $4.2 \mathrm{~K}$ ); and TAMU4, adding a double-pancake subassembly using high-performance superconductor. The short-sample fields in each case are 7.6 Tesla, 13.5 Tesla, and 14.1 Tesla. Note that in the case of TAMU4 the limit to field performance arises from the limit of $\mathrm{j}_{\mathrm{Cu}}<2000$ A during quench (limited in the outer layer). In TAMU2 we have located a probe coil at an accessible position, where the field strength at short sample should be 5.2 Tesla, in order to validate the transfer function.

Note the amount of superconductor needed in each dipole, expressed as the total cross section area of superconducting strand in the total windings. The $28 \mathrm{~cm}^{2}$ coil area of the optimizedconductor TAMU4 dipole is the least of any high-field VLHC design.

Table 1. Principal data for the coils of the TAMU dipoles.

\begin{tabular}{|c|c|c|c|c|}
\hline & TAMU2 & TAMU3 & TAMU4 & \\
\hline max bore field @ short sample limit & 7.6 & 13.50 & 14.05 & $T$ \\
\hline operating current & 10625 & 15.1 & 13.53 & kA \\
\hline stored energy & 0.062 & 0.478 & & $\mathrm{MJ} / \mathrm{m}$ \\
\hline $\max$ Lorenz forces & 0.93 & 6 & 9.9 & $\mathrm{MN} / \mathrm{m}$ \\
\hline \# windings & 2 & 4 & 8 & \\
\hline inner conductor: & ITER & $\mathrm{Nb3Sn}$ & Nb3Sn & \\
\hline \# strands & & 31 & 31 & \\
\hline strand diameter & & 0.773 & 0.773 & $\mathrm{~mm}$ \\
\hline Jsc & 1050@10T & 3000 & 3000 & $\mathrm{~A} / \mathrm{mm} 2$ \\
\hline Jcu & 1146 & 2790 & 2500 & $\mathrm{~A} / \mathrm{mm} 2$ \\
\hline \# turns & 11 & 13 & 4,13 & \\
\hline outer conductor: & ITER & $\mathrm{Nb} 3 \mathrm{Sn}$ & $\mathrm{Nb} 3 \mathrm{Sn}$ & \\
\hline \# strands & & 45 & 45 & \\
\hline strand diameter & & 0.54 & 0.54 & $\mathrm{~mm}$ \\
\hline Jsc & 1050@10T & 3000 & 3000 & $\mathrm{~A} / \mathrm{mm} 2$ \\
\hline Jcu & 1146 & 2790 & 2500 & $\mathrm{~A} / \mathrm{mm} 2$ \\
\hline \# turns & 16 & 22 & 22,22 & \\
\hline Total Area of SC & 3.34 & 16.63 & 28.02 & $\mathrm{~cm} 2$ \\
\hline
\end{tabular}


Load lines (Green lines areTAMU4, black lines are TAMU3, red lines are TAMU2)

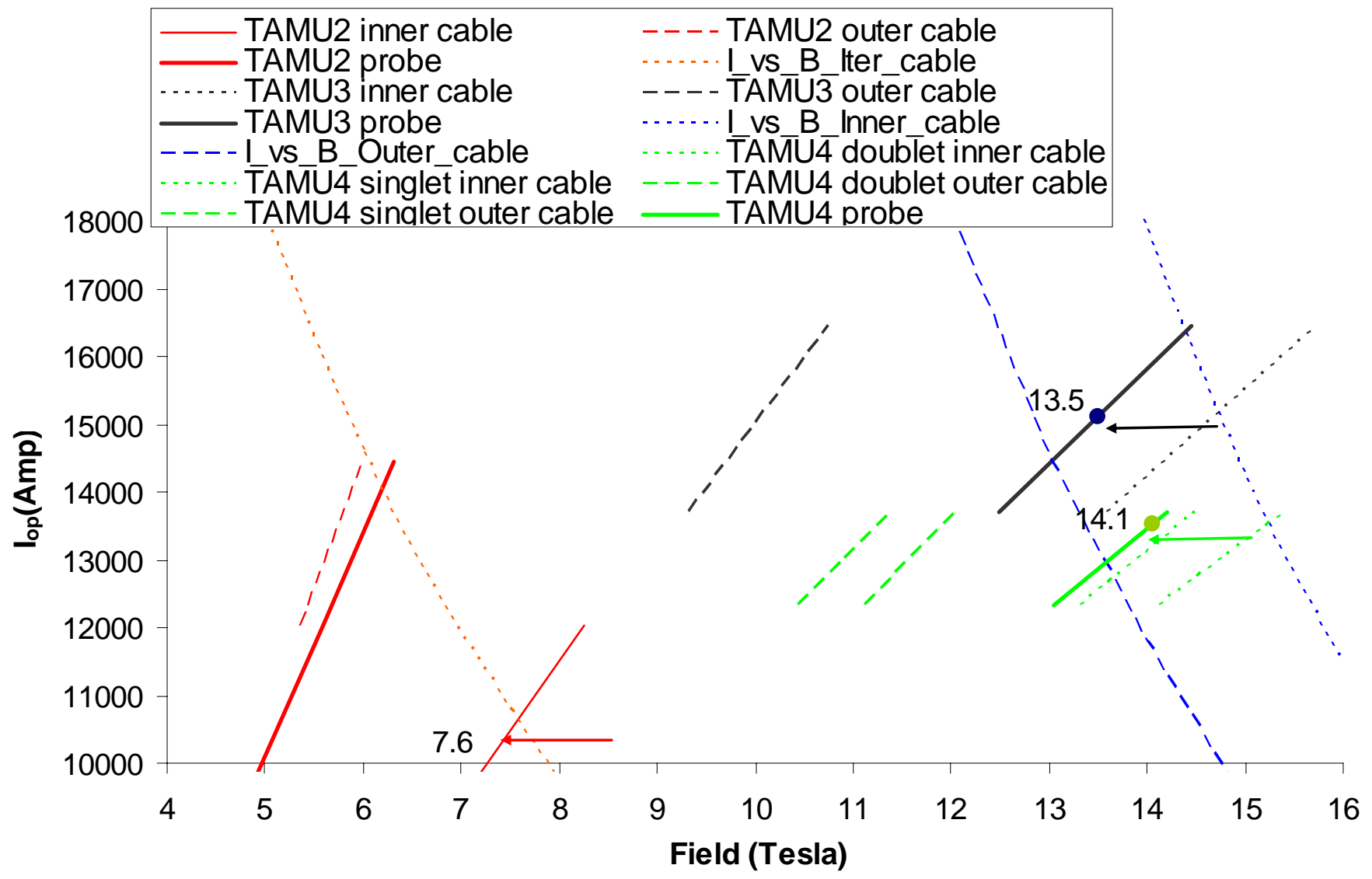

Figure 9. Load lines for TAMU2, TAMU3, and TAMU4. 


\section{Progress Report: Completion of TAMU2}

We have spent 2003-2004 building tooling and fixtures and developing fabrication techniques for TAMU2: winding fixtures and 10-stack tests of modulus and insulation; heat treat fixturing, gas flow manifolds; vacuum impregnation fixturing; expansion bladder systems; flux return half-assemblies; splice subassemblies and procedures; and fixtures for the support, transport, and manipulation of the dipole components through the entire sequence through completion as a dipole.

The year of 2005 was spent in actually building TAMU2. The best way to present what we learned during its construction is to talk about the successes we had in this first complete use of all fixtures and procedures, the problems that we encountered, and what we did to solve the problems.

\section{Provisions for module support and gas/epoxy flow manifolds in the thick skin}

The thick skin is the support framework that gives substance to each stand-alone single-layer coil module. We designed a matrix of flow channels into the thick skin to provide parallel flow of inert purge gas through the coil during reaction heat treat and of epoxy during vacuum impregnation. This manifold is shown in Figure 10.

The thick skin also contains a central channel that precisely locates and aligns the laminated Ti winding mandrel upon which the inner winding is wound and relies for preload.

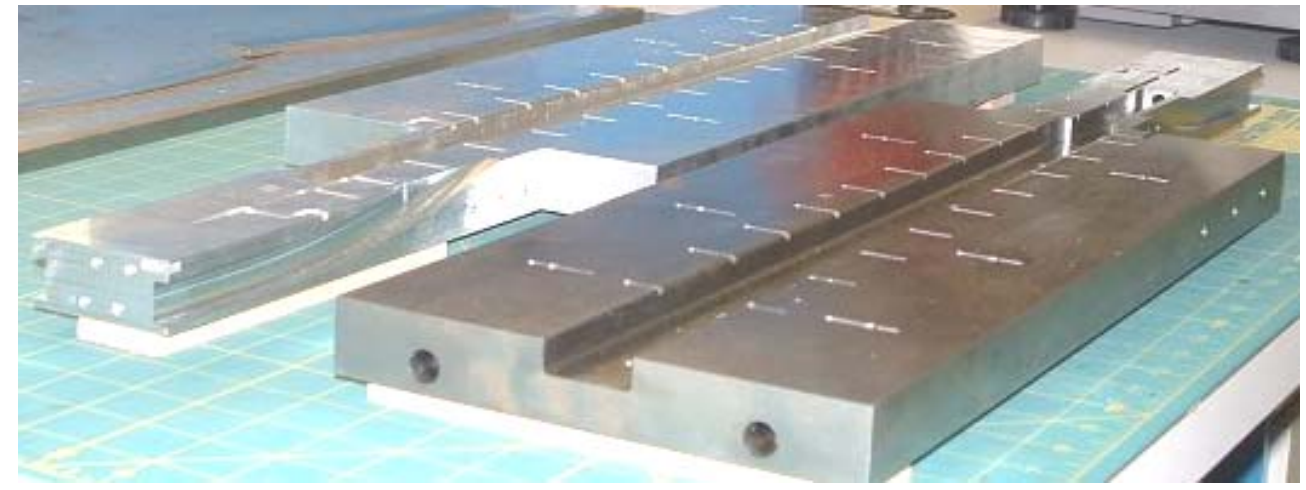

Figure 10. Matrix of flow channels in the thick skin.

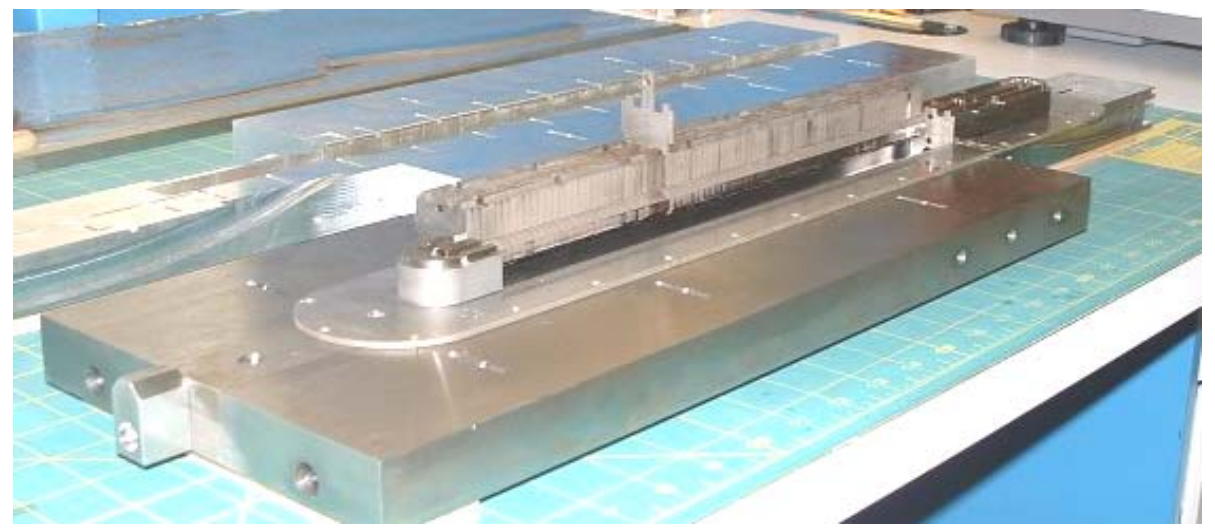

Figure 11. Laminated Ti mandrel being installed in slot in thick skin. 


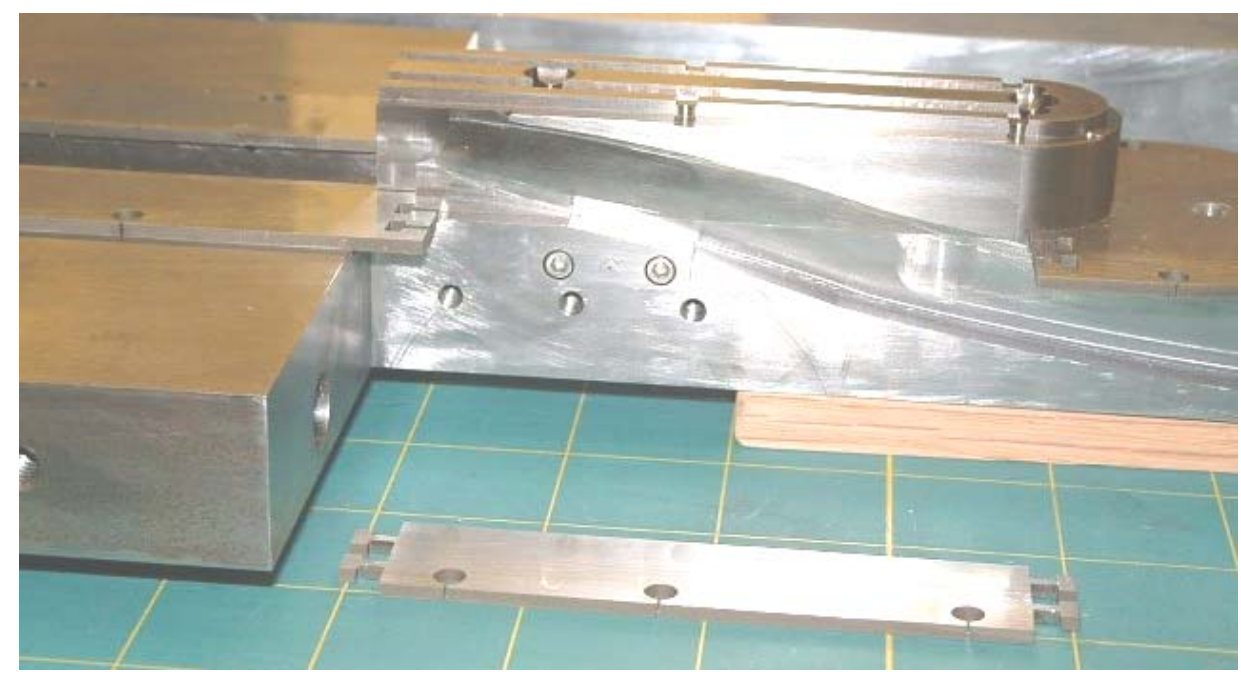

Figure 12. Coil side support sections and support section for one lead S-bend in thick skin.

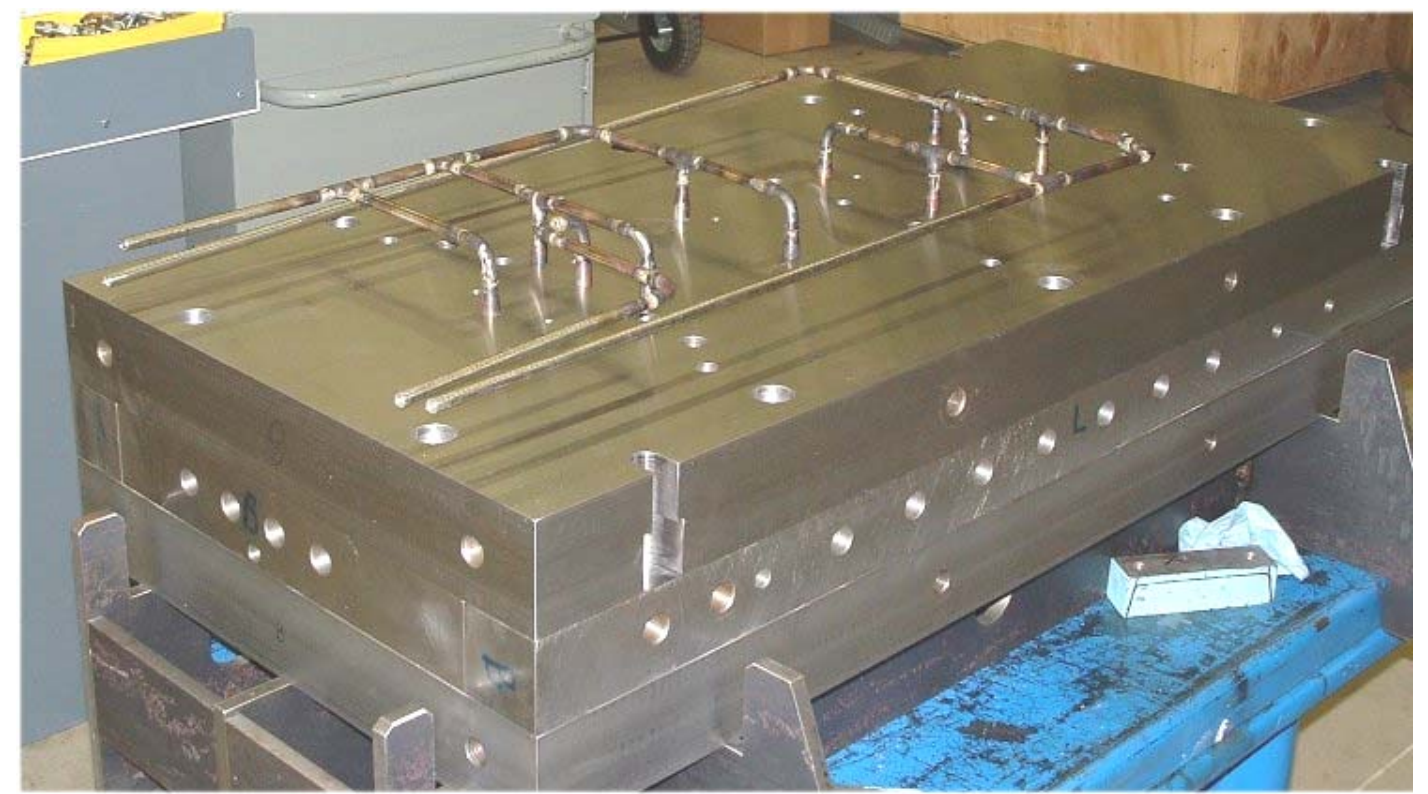

Figure 13. Coffin with lid in place and gas manifolds for Ar purge during reaction bake.

\section{Electrical Problems during coil winding}

Two electrical problems were encountered in the coil fabrication:

1) a low voltage short (finite conductivity) was encountered after the DI water rinsing procedure used to remove the acid flux that was used to remove the chrome coating from the ITER strand. This procedure will not be needed in future since it is only needed for removing $\mathrm{Cr}$ from ITER strand (there is no $\mathrm{Cr}$ coating on the highperformance internal-tin $\mathrm{Nb}_{3} \mathrm{Sn}$ wire that will be used in all future model magnets). The rinse water wicked up into the "S"-glass sleeve of the cable, depositing sufficient ionic debris to give $\sim 10-100 \mathrm{k} \Omega$ resistance to ground. This process is shown in Figure 14. 


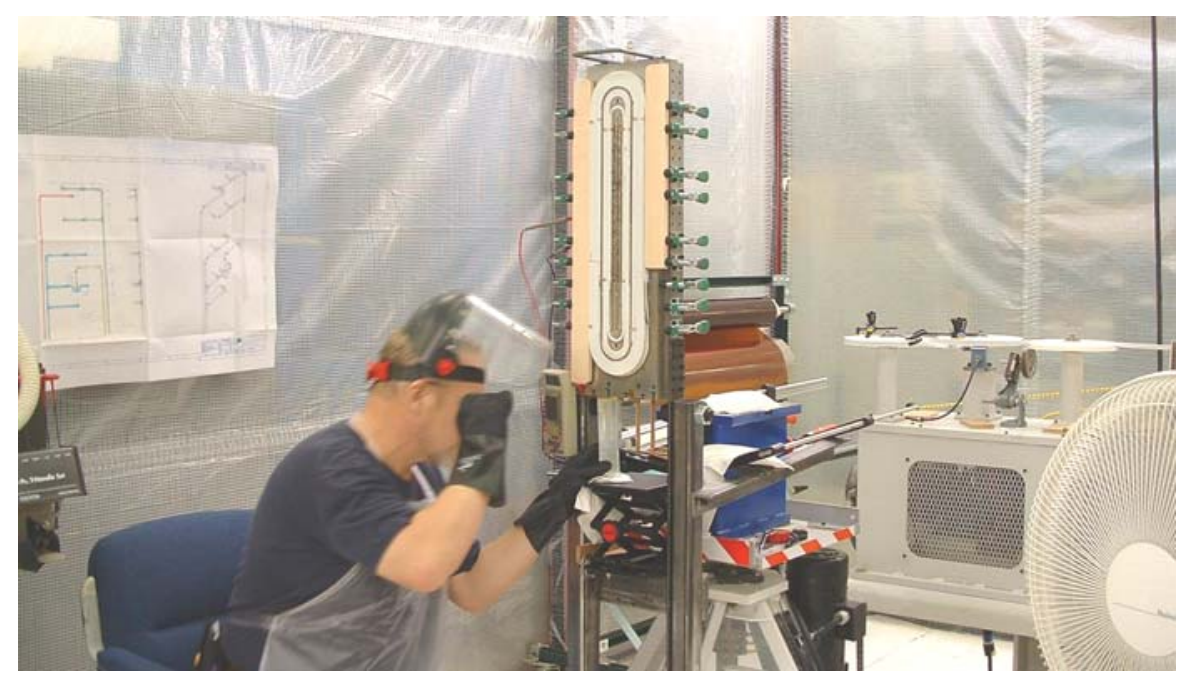

Figure 14. Acid dip process used to remove $\mathrm{Cr}$ from ITER cable in the leads.

2) We encountered a low hi-pot value of break-over voltage on the outer winding because of an abraded location in the S-glass insulation between the case and the return yoke. See comments in following discussion.

S-glass insulation: The original S-glass sleeve which was woven directly on the cable proved very robust to the winding of the actual coil. What little damage that appeared seemed to occur during the wash/drying cycle of the old sizing removal. After the removal of the original sizing, a new coat is applied by a set of nozzles which are located at the edges of the cable. This appeared to work very well with the exception when the line would have to stop palmitic acid application and drying of the sock (see Figure 8b). These stops would cause (if long enough) the sock to be discolored and the spray nozzles would sometimes clog up during the stops. These problems are presently being addressed by changing to an air brush applicator which will never be turned off. On the take up spool for the finished cable, a tape to separate turns that does not soak up the sizing (i.e. Teflon tape).

a) Problem with stop/start of the re-sizing line with uniformity of the cable color and sizing application as well as volume appears to be able to be reduced (this excess sizing volume is being wicked into the flat cable faces where it is not needed or wanted).

b) On the take-up spool for the insulated cable after new sizing has been applied use a material to separate the turns which will not remove sizing from the "S"-glass insulation by capillary action. (i.e. Teflon tape). The actual ITER strand cable used appeared to have very few "S"-glass filament bundles broken.

\section{Fabrication of laminar springs}

The coil pre-compression spring fabrication fixtures worked reasonably well with the exception of the curved spring welding fixture. We had great difficulty with getting the two edges of the spring close enough in proximity for the laser welding to be single pass. New modified fixtures will be needed for TAMU3 due to a slight cable size change. We will therefore make up all new fixtures even the straight spring and make them the full or slightly larger length than needed. The starting compression is about as large as can be accommodated, therefore if possi- 
ble it would be productive if the deflection for a given load were less and allow an increase in the preload of a few hundred psi.

\section{Material Specifications}

In order to achieve the dimensional tolerances and reproducibility that is required to construct the internal support structures, it has been necessary to single phase anneal the starting materials relieving their built in stresses. This is particularly true of the titanium and Inconel that the heat treatment sizing fixtures and internal support structure members of the coils are fabricated from. There appeared a problem with the internal support structures in the ITER strand cable windings which required a work-around repair and accommodation for a dimensional change after it had gone through the reaction bake (see Figure 15). One of the more difficult materials was the titanium plate used in the winding mandrill or post as a welded structure composed of laminations. After encountering this problem we performed a thorough set of dilatometry studies of the materials (Inconel, Ti, A15 steel) that compose the winding assembly and determined that the dimensional changes were the result of the release of rolling strain in the materials during heat treat, and that the effect could be eliminated by stress-relieving the materials prior to machining. The affected parts on TAMU2 were replaced and no damage was evident.

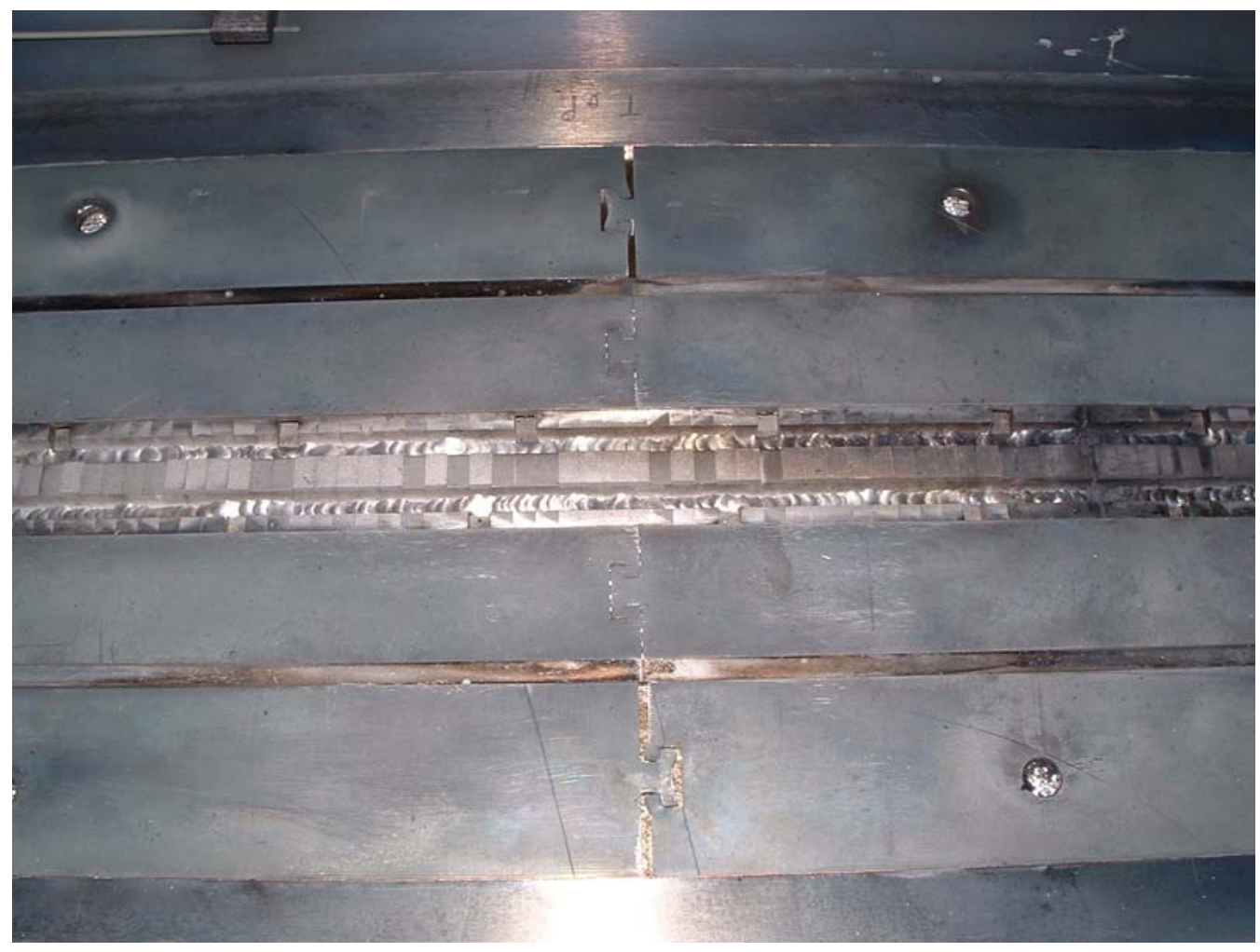

Figure 15. Damage to stress management containment structure due to release of pre-strain during heat treat. 


\section{Fixtures for heat treatment of single-pancake winding assembly}

Argon is purged through the coil during heat treat to remove gases and vapors evolved from decomposition of remnant sizing and surface contaminations. Two independent argon purge flows are provided. The interior Ar flow passes through the coffin/windings and discharges through a filter. The flow passes in parallel through the inner and outer windings. The gas flow through each path is separately controlled and monitored by a downstream valve and bubble monitor (see Figure 16). A second independent flow is provided from the inside to outside of the kiln and from there to the exhaust through a valve and bubble monitor.

It was found that the three zone furnace heat control was adequate to control the reaction heat treatment cycle of the $\mathrm{Nb}_{3} \mathrm{Sn}$ conductors according to the data recorded by the thermocouples located next to the windings inside the coffin. We provided separate parallel gas flows for the purge flow inside the coffin (winding sizing fixture) and for the ambient atmosphere in the retort. The two flow rates in these two regions were separately adjusted to provide a purge through the coil and a clean retort atmosphere.

The overall area for the heat treat furnace and the vacuum impregnation system is shown in Figure 17. We also re-worked the support gantry and lifting mechanisms on the heat treat furnace to stabilize them for safe operation and smooth travel in lowering the dome onto the stack of heater elements. Figure 18 shows the base of the furnace with the magnet inside its coffin in place for reaction bake, prior to lowering the outer shell.

The same coffin fixture is used to support the coil assembly in both reaction bake and in vacuum impregnation. The coil assembly is removed from the coffin after reaction bake and the coffin is cleaned and refitted for use in vacuum impregnation (Figure 19). Figure 20 shows the coil placed inside the refurbished coffin, with the gas purge connections for heat treat being completed. 

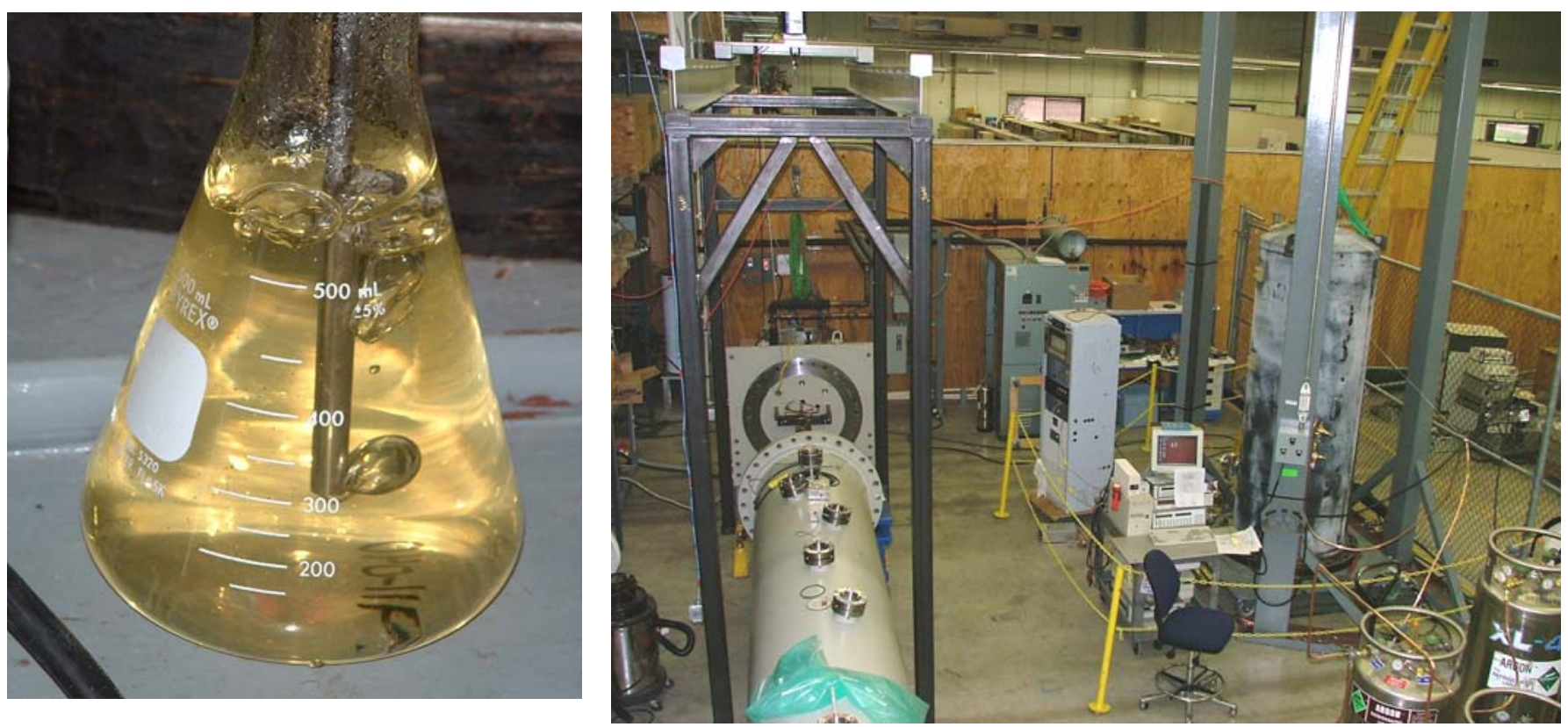

Figure 16. Bubbler samples sizing contamination in purge gas during reaction bake: the windings of TAMU2 were very clean.

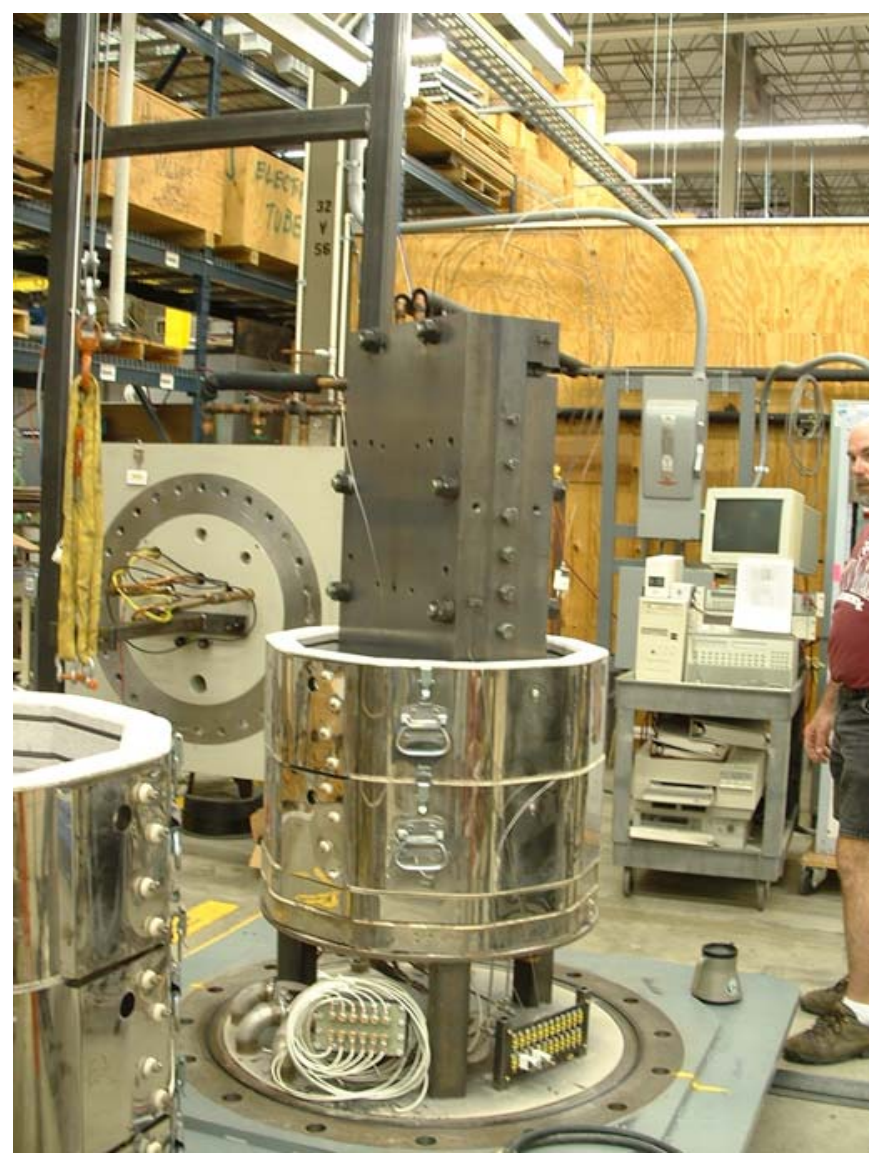

Figure 17. Overview of reaction bake furnace (room-temp dome to right) and vacuum impregnation retort (left) with gantry.

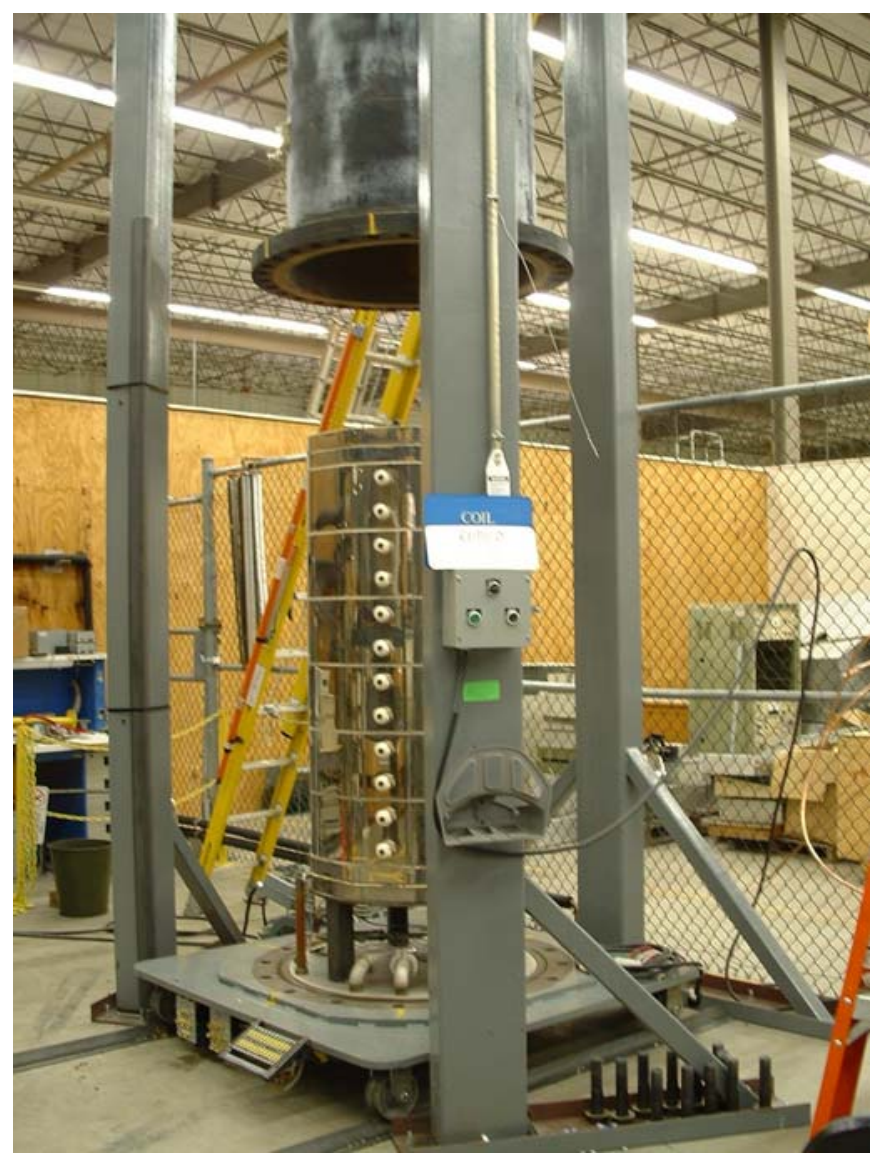

Figure 18. Two stages of assembly of the magnet in its support coffin onto the base of the heat treat furnace. 

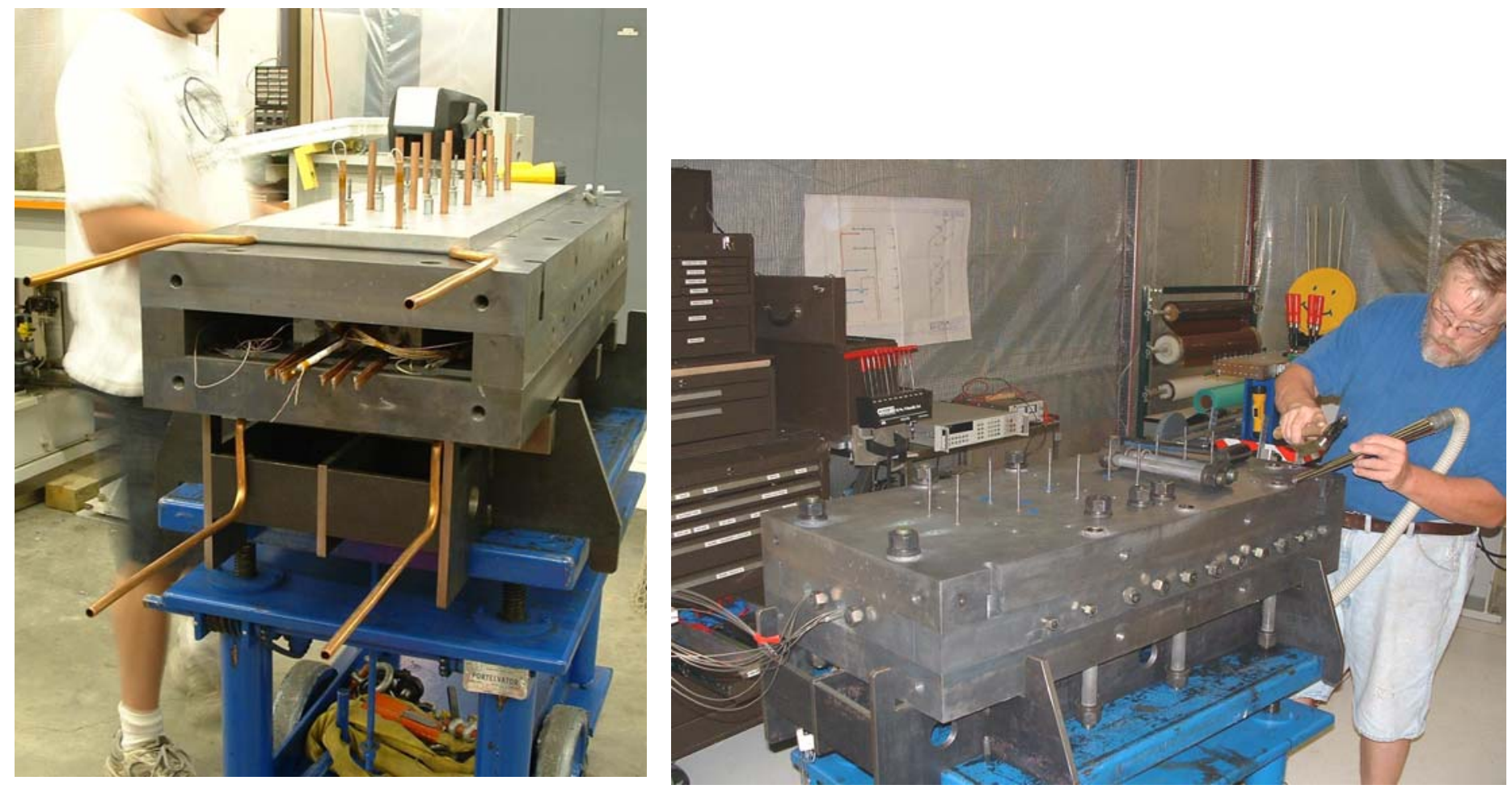

Figure 19. Preparation of coffin after reaction bake, ready for re-use to support coil assembly during vacuum impregnation.

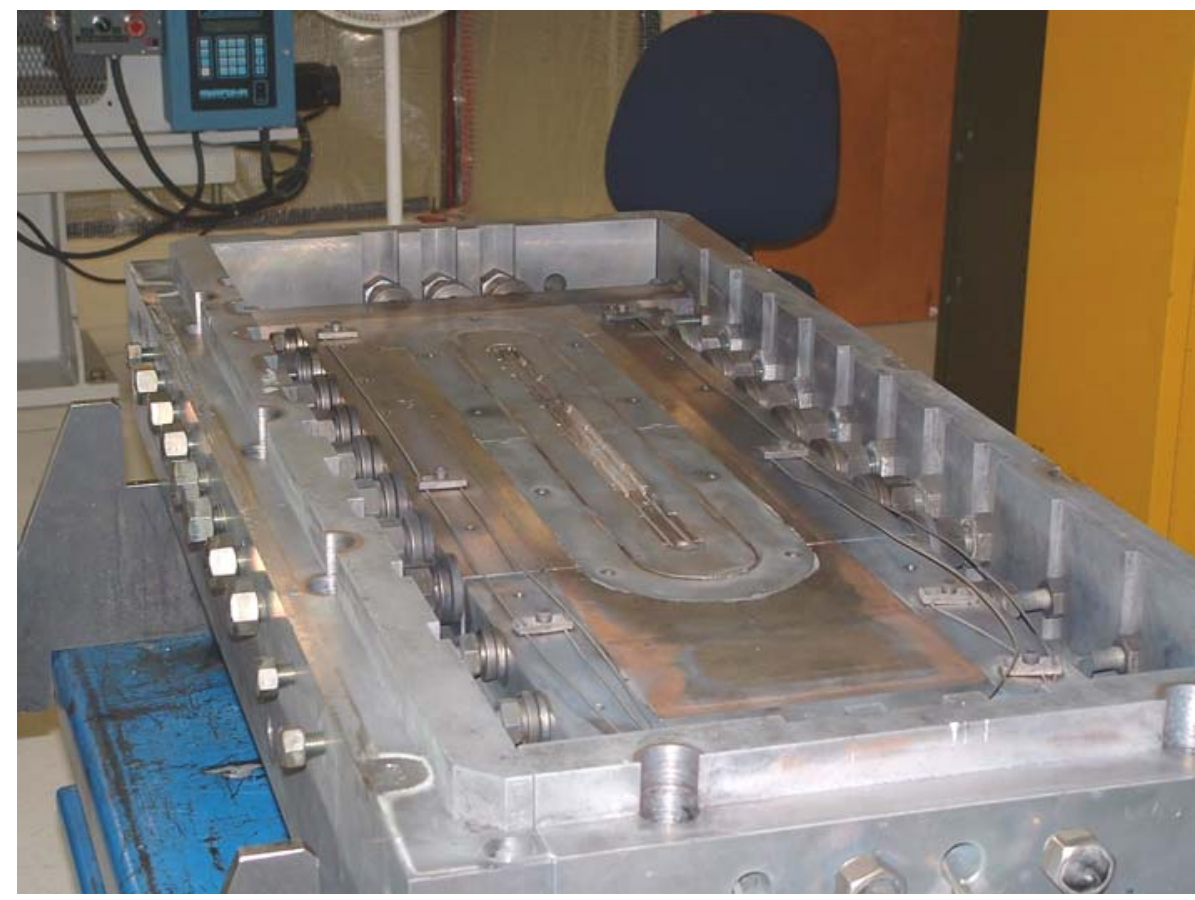

Figure 20. Preparation of coffin and coil assembly for impregnation. 


\section{Vacuum Impregnation System}

We re-worked a number of elements in the vacuum impregnation system to enable us to have full control of epoxy flow during filling, hot-oil heated coaxial supply and return lines so that epoxy cannot thicken while feeding to the coil, and improved visibility and internal lighting within the retort so that we can see all feed pots and the magnet overflow cups within the retort. A new overhead hoist (Figure 18) was installed which can service the coffin either from the heat treat kilns or the impregnation chamber.

We found in the impregnation of our last dipole (TAMU1) that the rapid access that the rollback retort provides can prove crucial in being able to intervene when something goes wrong. This time we have improved the epoxy flow provisions as well. A magnet carrier was built to transport the magnet in and out of the impregnation retort, and oil-heating channels for epoxy injection lines were added to the vacuum retort (Figure 21).

At the time of our last renewal proposal the coffin was being removed from the heat treatment furnace. The next step was to prepare the reacted winding for impregnation. Those steps include: the wiring of the coil; installing voltage taps, protection heaters, and Kapton/stainless steel composite ground plane foil; recording any discrepancies in the coil visible at this stage in the preparations and fixing any observed problems if possible.

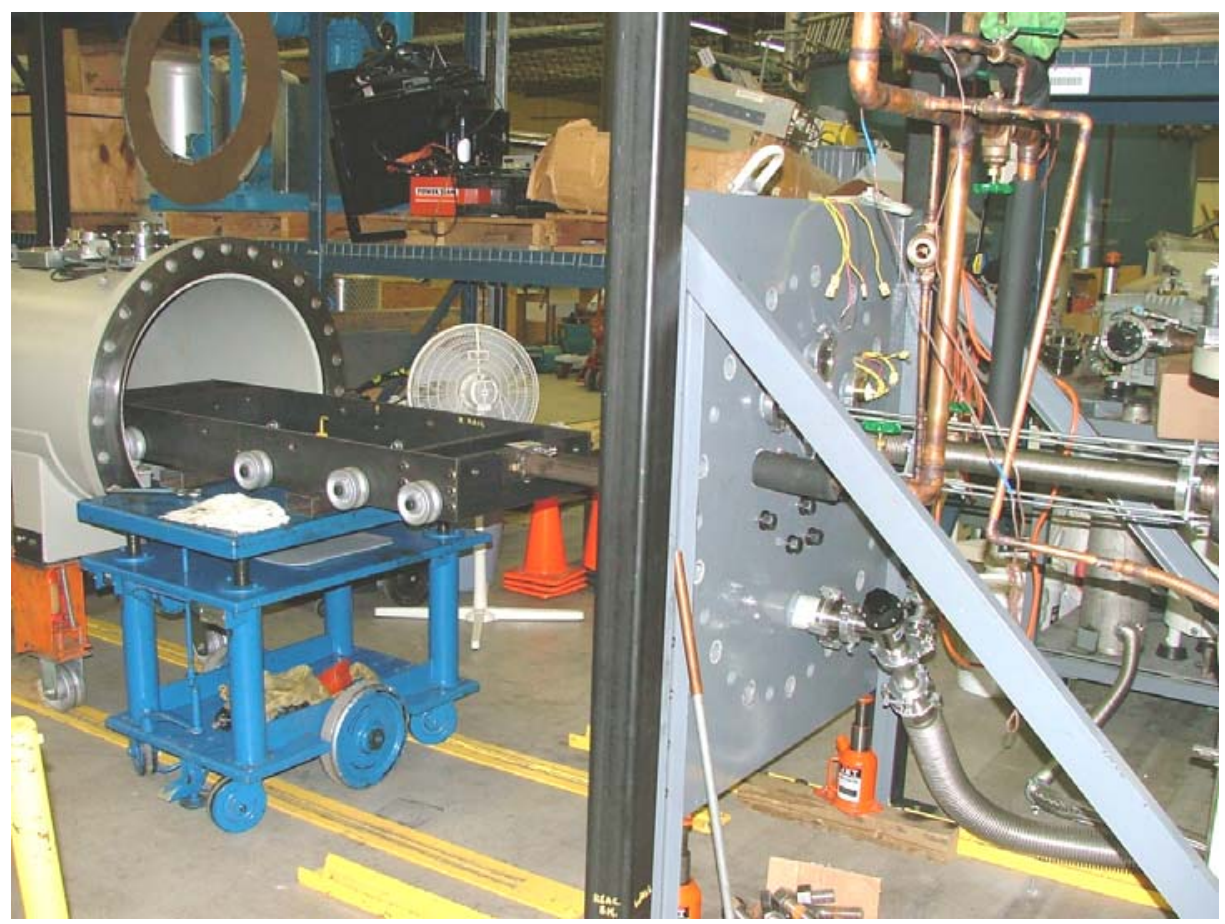

Figure 21. Support trolley for coffin, positioned in the entrance aperture of the vacuum impregnation system. End plate with hot oil supply and return lines for the heated epoxy supply line. 
The epoxy impregnation system: worked very well in practice but used a fairly large volume of CTD101. After the cure cycle was completed, an inspection of the chamber revealed the majority of it in a drip tray at the lead end of the fixture. This loss was traced back to where the leads left the fixture, which is on am level above the winding but not the overflow cups. There are several better ways in which to seal this NbTi cable afterwards which will be pursued during the preparations for the TAMU3 impregnation. There were no heating/cooling oil circuit leaks detected and no leaked oil was found in the fixture, chamber or outside during disassembly. The temperature controller on the circulating heating/cooling oil stream was replaced by a newer style controller with a remote option, but in practice we wired that out to get even better stability and it worked very well. It would however be nice to have a more modern user-friendly unit.

Figure 23 shows the coil module after impregnation. 

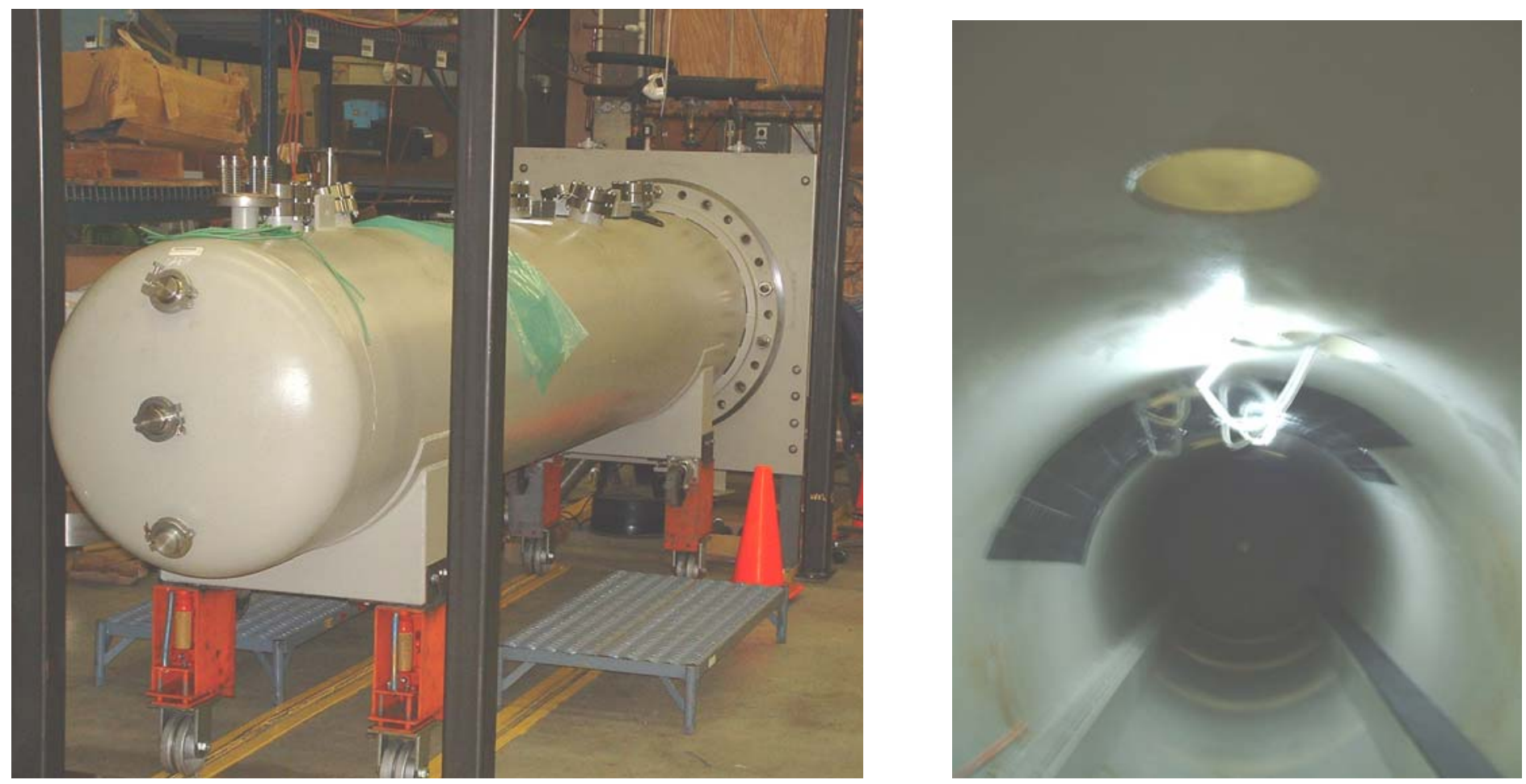

Figure 22. a) outside of coffin/coil vacuum chamber impregnation fixture; b) inside of the vacuum vessel for coffin/coil impregnation fixture.

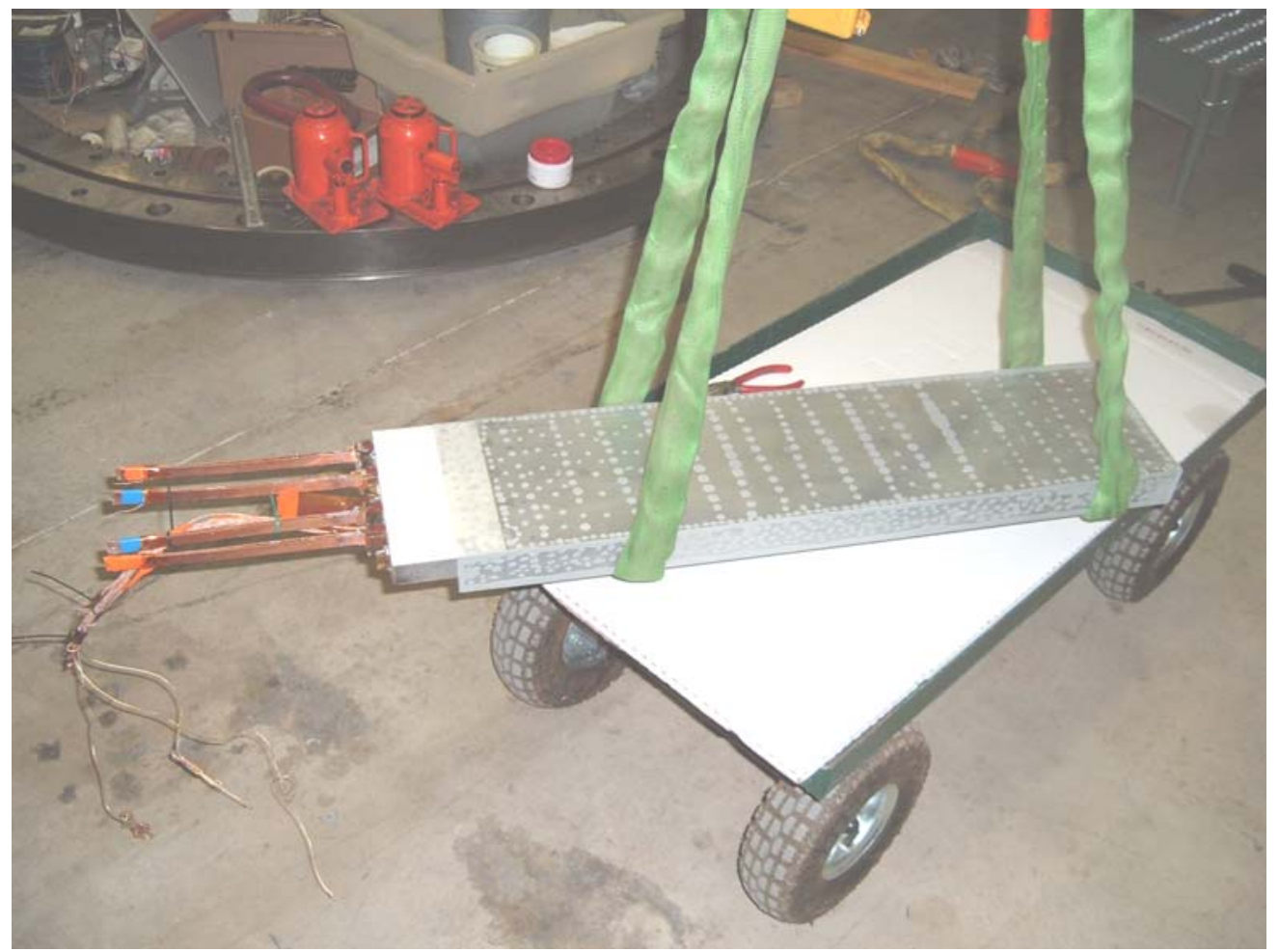

Figure 23. The finished coil module (Kapton-insulated NbTi leads extend from splice supports). 


\section{Return iron yoke and aluminum stress tube}

The cylinder was shortened by two inches in order to simplify the adaptation fixtures needed in order to use the LBNL vertical test Dewar for the TAMU2 testing. The only problem that was encounter using the handling procedures presented in Appendix A is that the threads on the nitronic rods were specified too close. This meant that when the rods were stretched, the threads did too even more relatively, thus making it impossible to engage the nut far enough down the threaded rod to contact the stainless steel half end plate surface.

\section{Bladder fill / pressurization system}

There was a third larger volume circuit added to the other two solder fill/pressurize circuits. Each circuit is a stand alone, with its own volume of solder and pressurization system that can be adjusted independent of the other two circuits. The connecting line to the bladder inlet valves are heated co-axially with hot water. The valve and bladder connecting lines are heated by Al heater blocks which contain thermocouples and electrical cartridge heaters. During the preliminary fill/pressurize cycles, variacs were used to control the temperature of these heaters, but that scheme was found not to give the response time of control needed. The actual winding module fill/pressurization cycle for the bladders used thermocouples at the coolest spot and temperature controllers. This combination gave the control sensitivity needed. It was also found that the connecting lines had to be flexible to a degree in order not to torque the bladder inlet lines which lead to a bladder failure.

It was noted that, with the horizontal fill of the bladders, several minutes elapsed before the solder level in them came to equilibrium (level) down the axial length of the magnet assembly. In the first experimental trial of the two bladder circuits, we observed variations in the texture of the solder fill, indicating that the end of the assembly nearest the fill lines cooled first so that the still-liquid far end may have solidified without hydraulic preload. To prevent this happening for preloading TAMU2, we sectioned the cooling jacket into four axial segments, shown in Figure 25 , and provided cooling water flow control so that we can initiate solidification at the end farthest from the fill lines and control the progression of the freeze-front so that it moves laminarly towards the fill lines. Upon disassembly of TAMU2 we will attempt to extract one bladder early before it melts and check its texture to verify the fill density.

The azimuthal bladders were filled and pressurized with the yoke/tube assembly in a vertical orientation (Figure 25) in order to attain cylindrical symmetry in the location of the flux return within the Al stress tube. The vertical and horizontal bladders bordering the coil assembly were pressurized with the dipole assembly in the horizontal position (Figure 26) for better coil positioning. The pressure in the azimuthally bladders was found to be $\sim 200 \mathrm{psi}$ low when checked horizontally. 


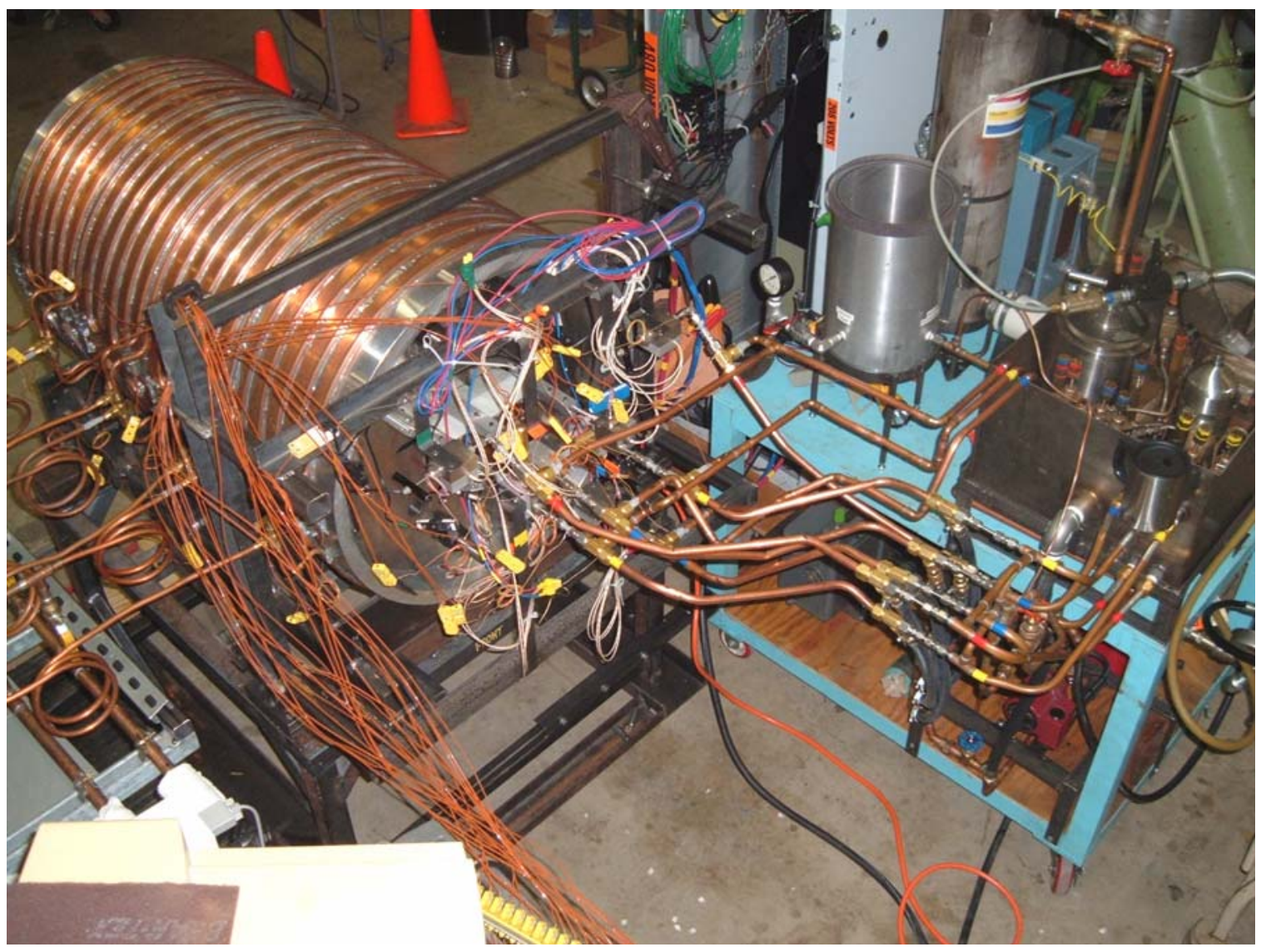

Figure 24. The hydraulic system for filling and pressurizing the expansion bladders.
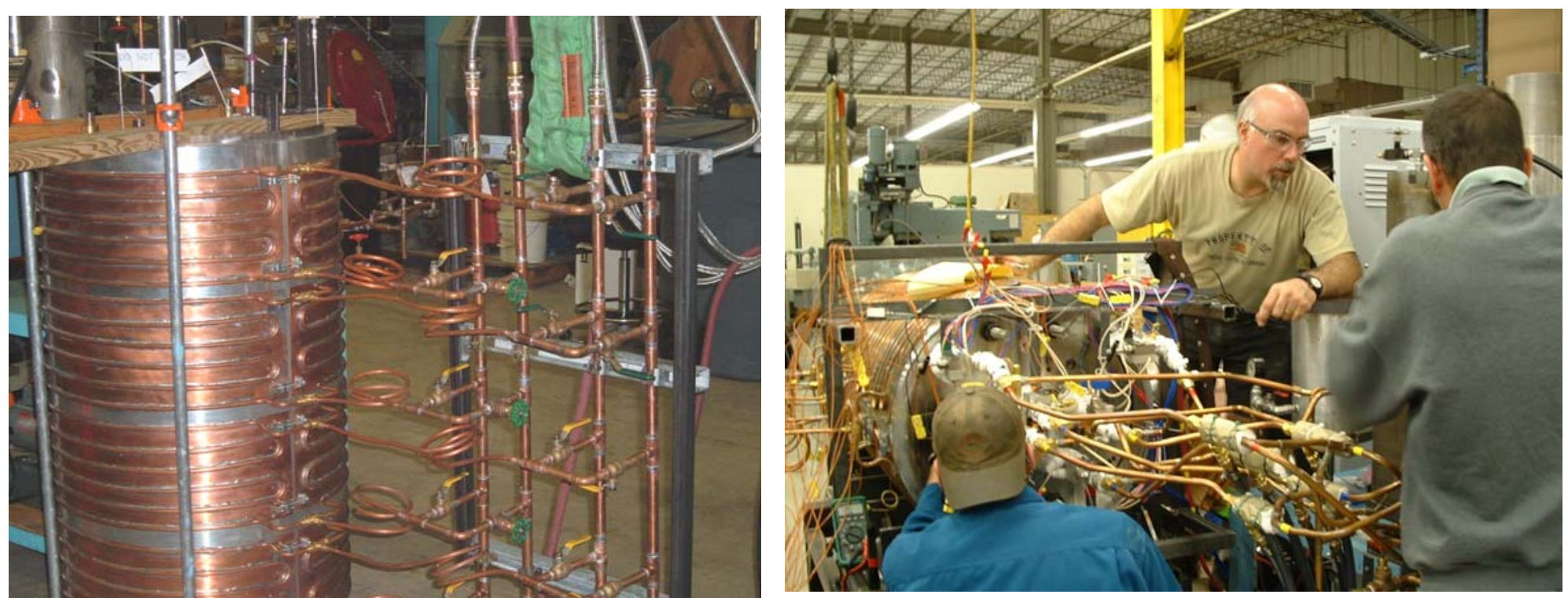

Figure 25. Zoned cooling for bladder pressurization.

Figure 26. Bladder pressurization in action. 


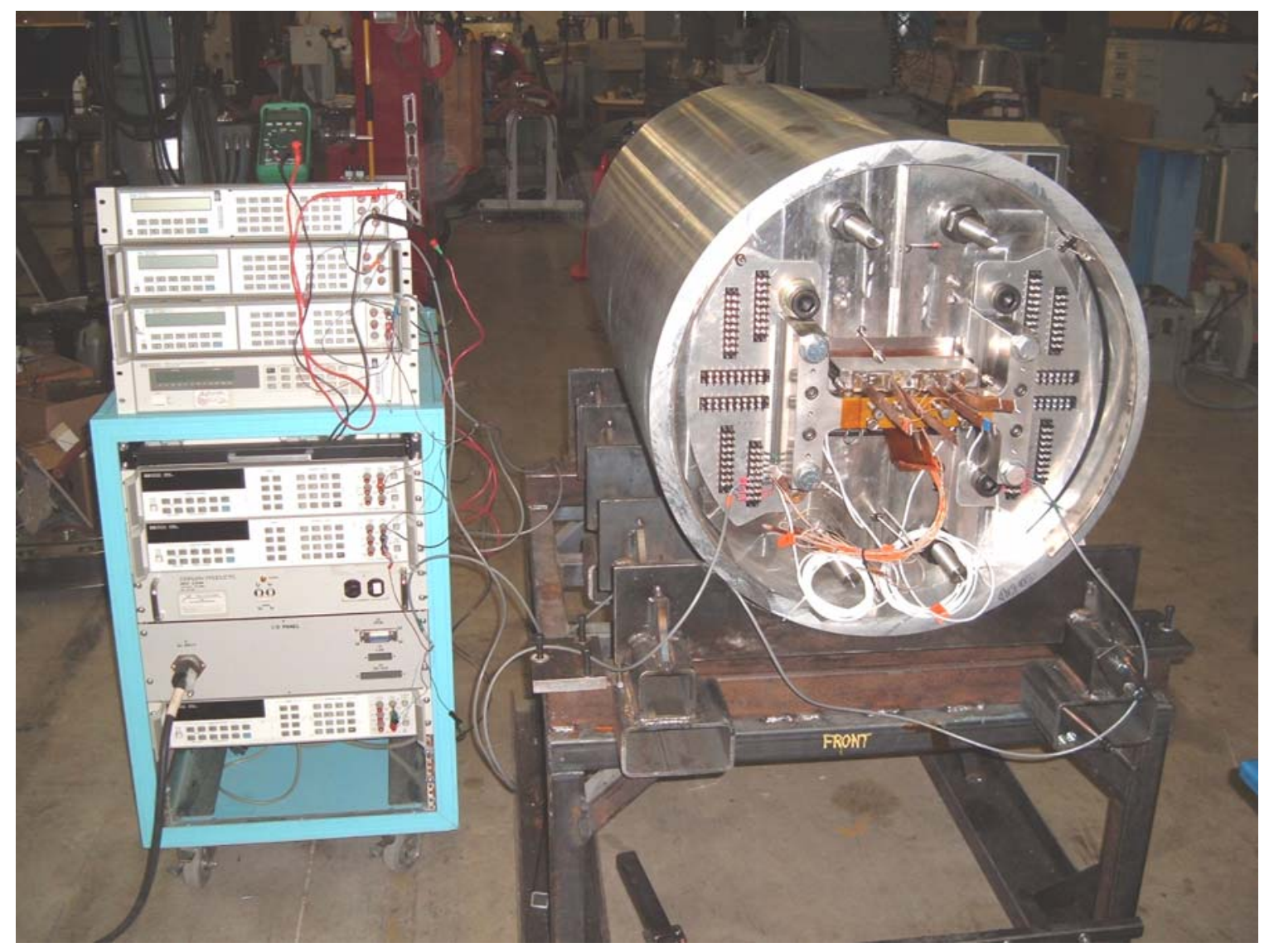

Figure 27. The completed TAMU2 dipole, with lead connections and instrumentation ready for connector wiring and testing.

\section{Preparations for testing TAMU2}

TAMU2 has been shipped to LBNL where it will be cold-tested. The new LBNL cryostat requires a short extension in order to accommodate the length of the overall TAMU2 assembly. We built a spacer ring for this purpose, consisting of an aluminum ring $1 \mathrm{~m}$ diameter and $\sim 3.5$ " thick which accepts through bolts from the cryostat flange pattern and has an "O" ring groove in the upper surface with the same dimensions as the one on the cryostat. 


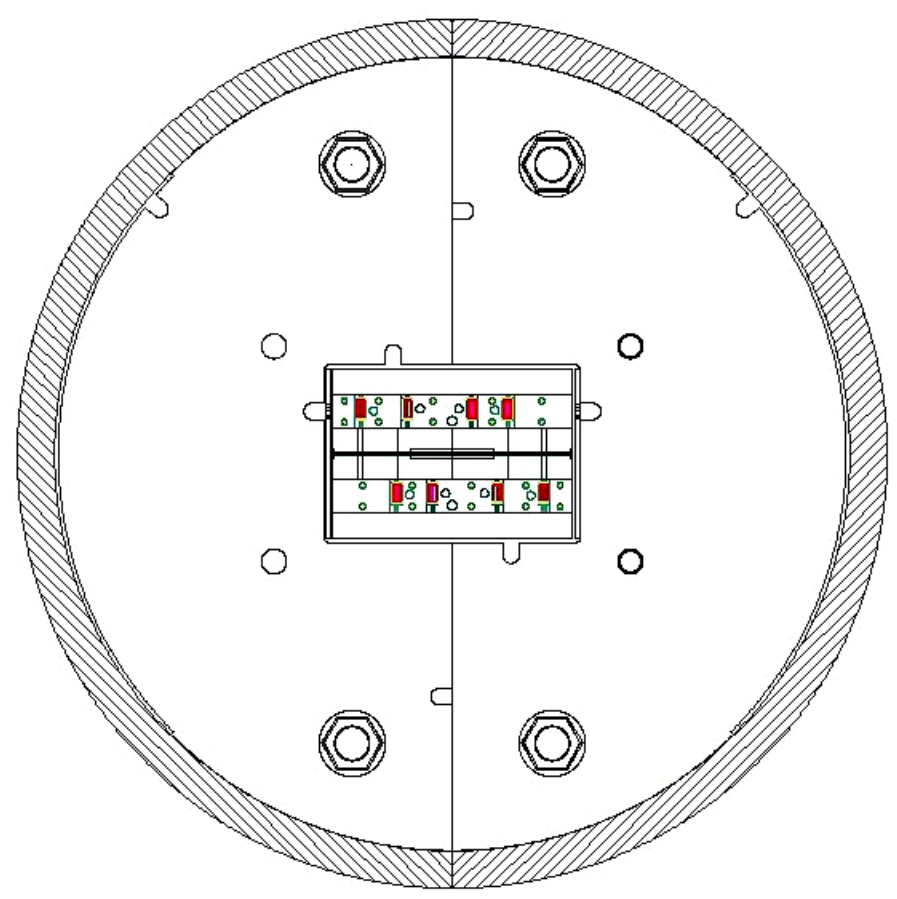

Figure 28. TAMU3: Two single outer pancake coils, yielding $13.5 \mathrm{~T}$ short-sample limit.

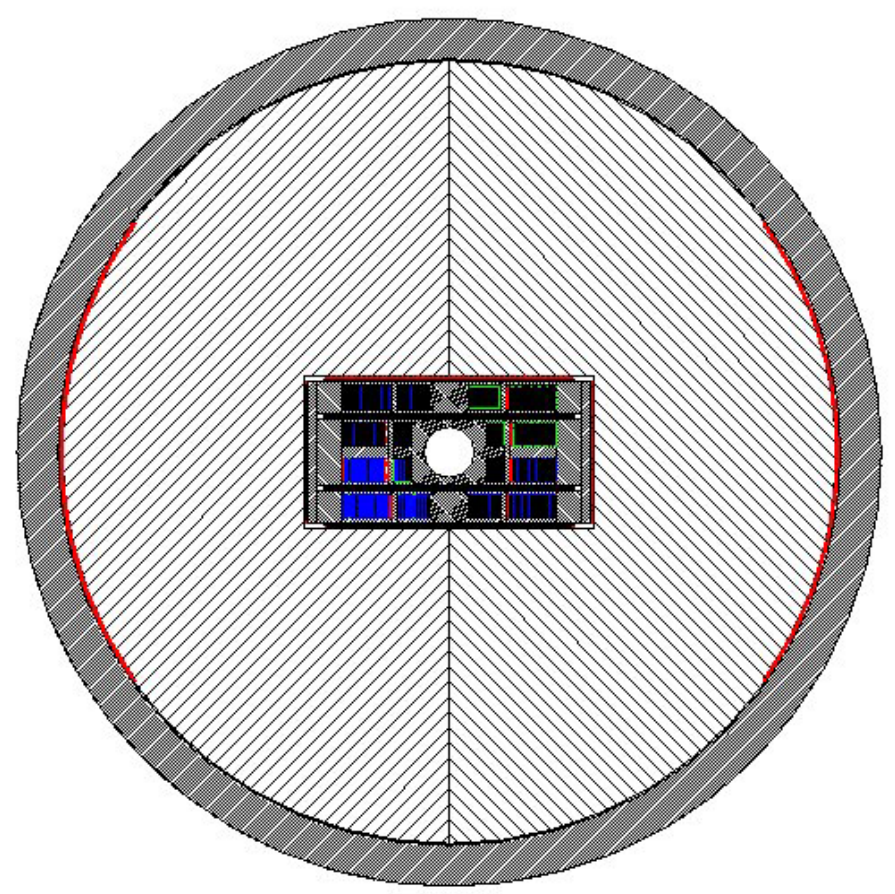

Figure 29. TAMU4: full embodiment of the 14 Tesla collider-capable dipole. 


\section{Contributions of the TAMU program to LHC commissioning and upgrade}

As we develop this high-field $\mathrm{Nb}_{3} \mathrm{Sn}$ dipole technology, it has led during the current year to four innovations that are directly pertinent to the commissioning and upgrade of LHC:

- An electrode assembly, suitable for integration within the existing LHC dipoles, capable of killing the electron cloud effect - an effect that threatens to limit the luminosity that could be attained in LHC;

- $\mathrm{A} \mathrm{Nb}_{3} \mathrm{Sn}$ structured cable, which makes it possible to design very high gradient quadrupoles for upgrade of the interaction regions of LHC to enhance its luminosity;

- $\quad \mathrm{A} \mathrm{Nb}_{3} \mathrm{Sn} / \mathrm{NbTi}$ levitated-pole dipole for use in the D1 bends that combine and separate the beams at the intersection regions. The levitated-pole design uniquely solves the problems of radiation damage and heating from particles swept from the beam.

- A hybrid dipole technology, in which inner windings of Bi-2212 are integrated in a $\mathrm{Nb}_{3} \mathrm{Sn}$ block-coil dipole to push to 24 Tesla, opening the possibility of a future tripler upgrade of LHC.

The following discussion summarizes these four innovations. We are in active discussion with CERN concerning all four concepts. None of the innovations will be developed under the proposed renewal of our base program, which is totally focused on making TAMU4 a success. We plan to submit separate proposals for funding of each of these innovations to DOE programs appropriate in each case. 


\section{Electrode assembly to kill electron cloud effect in the LHC arc dipoles}

In Spring 2005 Prof. McIntyre spent a month at CERN, working with the AT division who are coordinating the construction, testing, and installation of the superconducting magnets. He found that there was general agreement that the electron cloud effect poses serious concerns for cryogenic heat load and for emittance growth, yet there was no plan to try to kill the effect in the arcs that constitute the bulk of the circumference. The electron cloud effect is a regenerative mechanism, illustrated in Figure 30. Electrons are liberated when a bunch passes, both by direct ionization of the residual gas and by photoionization from synchrotron light. The electrons have thermal energy and so are still in the vacuum when the next bunch passes. They are accelerated by the Coulomb field of that bunch, attain $\sim \mathrm{keV}$ energy, then strike the beam screen wall and release secondary electrons with a yield $h$ which for copper varies from $\sim 1.1$ to 2 .

This scenario provides a regenerative mechanism by which a large static charge can accumulate, producing a cryogenic heat load to the beam screen and dynamic interaction with bunches leading to instabilities and emittance growth. Both effects can pose significant limits to bunch intensity, bunch spacing, cryogenic loading, and luminosity.

We have devised an electrode assembly ${ }^{14}$ that can kill the electron cloud effect by presenting a continuous $+100 \mathrm{~V}$ clearing electrode along the bottom of the beam screen. A full-length prototype is being installed and tested in an LHC arc dipole this summer. Pending evaluation, CERN will need to decide whether they want the electrodes installed in all dipoles. If the answer is yes, the TAMU group will propose to coordinate the construction and installation of the electrode assemblies as a US contribution to the commissioning of LHC. This role has the potential to eliminate an effect that could otherwise pose a severe limit to LHC luminosity.

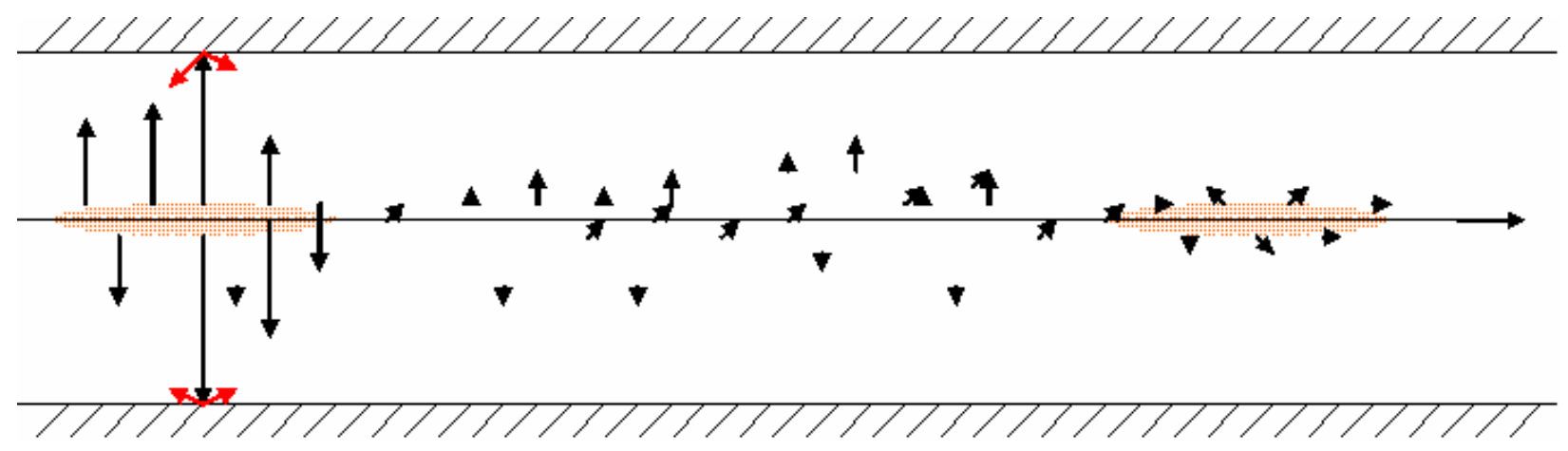

Figure 30. Schematic illustration of electron cloud effect.
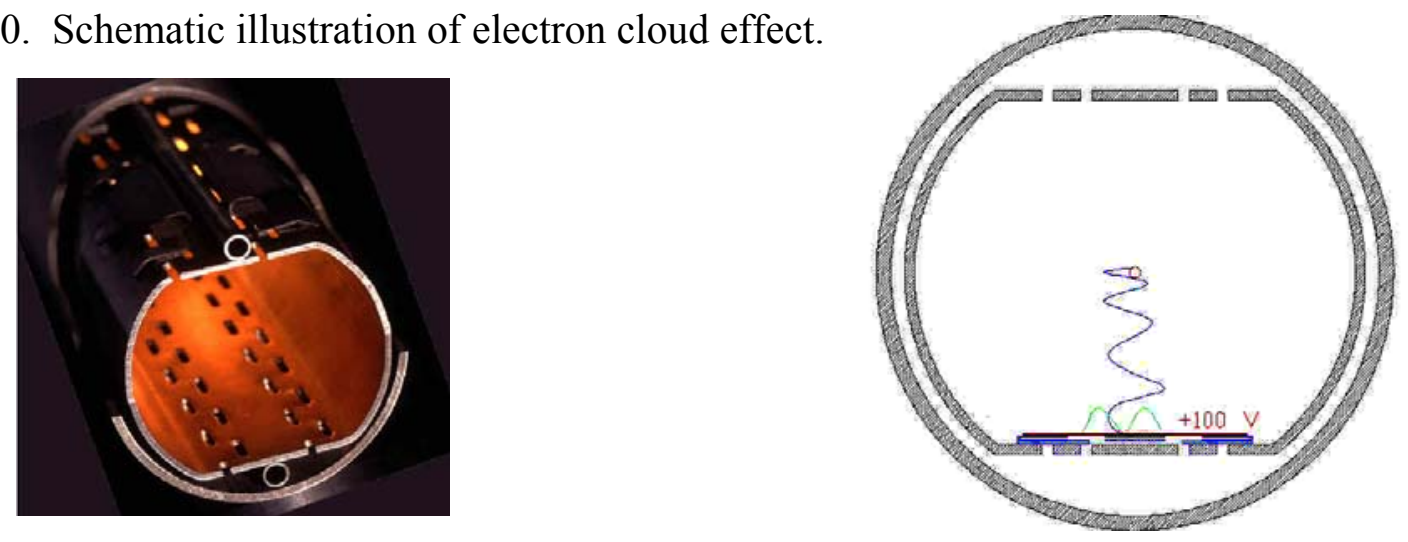

Figure 31. a) beam screen of LHC dipole; b) electrode assembly installed in beam screen. 


\section{Structured cable quadrupoles for Q1 quadrupoles in LHC luminosity upgrade}

The most effective way to enhance luminosity in a hadron collider is to move the quadrupole triplet as close to the intersection point as possible. This is exemplified in the intersection regions (IR) at CDF and D0 at the Tevatron, but has not been done for LHC because of concerns about the ability of the quadrupoles and dipoles to operate with the fluence of particles expected at LHC luminosity.

We set out to resolve this limitation by devising a quadrupole that could accommodate very large loads of heat and radiation damage from particles streaming into its face in a short-baseline $\mathrm{IR}^{15}$. For this purpose we propose to develop a $\mathrm{Nb}_{3} \mathrm{Sn}$ version of a structured cable that we developed some years ago for use with Bi-2212 superconducting strands ${ }^{16}$. The structured cable has three provisions that make it ideal for the application:

- It integrates mechanical support of the superconducting strands at the cable level, so that stress does not accumulate upon the strands of succeeding cable layers in the coil;

- It provides a cooling channel within each cable element, which can be opened at the ends to provide parallel-flow cooling with superfluid helium, dramatically enhancing heat transfer;

- The round cable geometry makes it possible to design for high gradient and small aperture with maximum flexibility for killing undesirable multipoles;

- The resistive sheath on the structured cable can provide sufficient guidance of supercurrents so that no insulation is required between adjacent cables, removing a weak element for radiation damage and providing for stable, low-voltage behavior during quench.

Figure 32a shows a cross section of the $3 \mathrm{~mm}$ diameter cable. Figure $32 \mathrm{~b}$ shows one quadrant of a $40 \mathrm{~mm}$ aperture quadrupole using this cable to produce $350 \mathrm{~T} / \mathrm{m}$ in $6 \mathrm{~K}$ superfluid He.

We plan to seek funding from the LARP program to develop the structured cable and coil technology appropriate for the quadrupole application.
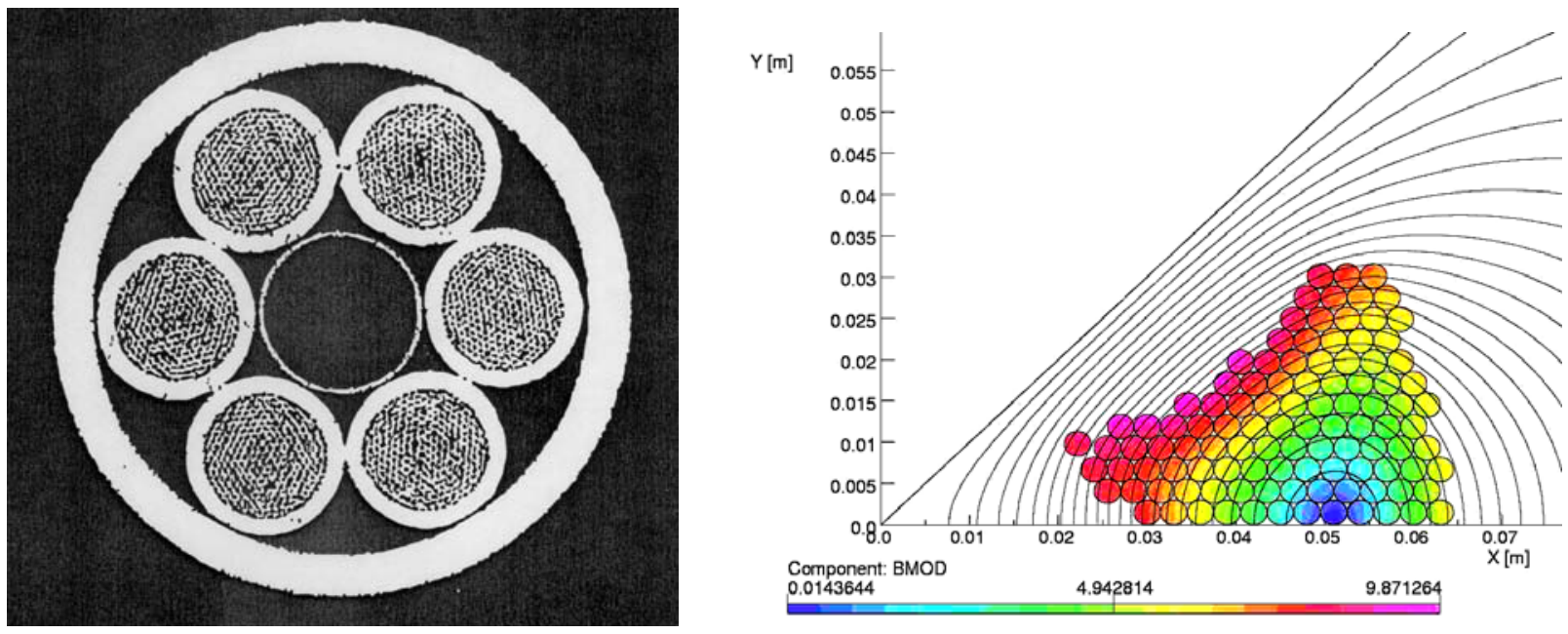

Figure 32. a) Structured cable containing 6 strands around spring core and outer sheath; b) cable elements in one octant of a quadrupole, designed for $350 \mathrm{~T} / \mathrm{m}$ at $6 \mathrm{~K}$. 


\section{Levitated-pole dipole for DI dipole in LHC luminosity upgrade}

The dipoles D1 combine and separate the beams in the IR. One third of all particles produced in p-p collisions pass down the beam tube into these dipoles; those particles carry two thirds of all collision energy, and they are all swept out of the beam tube by the dipole field into the side walls of the dipoles. The heat and radiation damage from this particle flux make a seemingly impossible challenge for the use of superconducting dipoles for the purpose.

We have devised a levitated-pole dipole that can accommodate the heat and radiation damage while providing an $8 \mathrm{~T}$ dipole field suitable for the separation geometry ${ }^{15}$. The flat-pancake windings are bonded to a pair of cold-iron pole pieces that are suspended within a warm-iron flux return, as shown in Figure 35. By proper placement of the insulating vacuum gap interface between pole piece and flux return, the Lorentz forces between coils can be made to cancel the image forces at the (unsaturated) steel at the interface. The pole pieces can then be supported against gravitational load and destabilizing forces using low-heat-load tension supports.

The important benefit of this design is that the side regions are completely open: particles from beam-beam interactions are swept into a $45^{\circ}$ aperture and first strike a warm-iron wall. This resolves the problems of heat load and radiation damage. The net effect of using the levitated-pole dipole for D1 and the structured-cable quadrupole for Q1 is shown in Figure 34.

We plan to propose funding from the LARP program for development of D1.

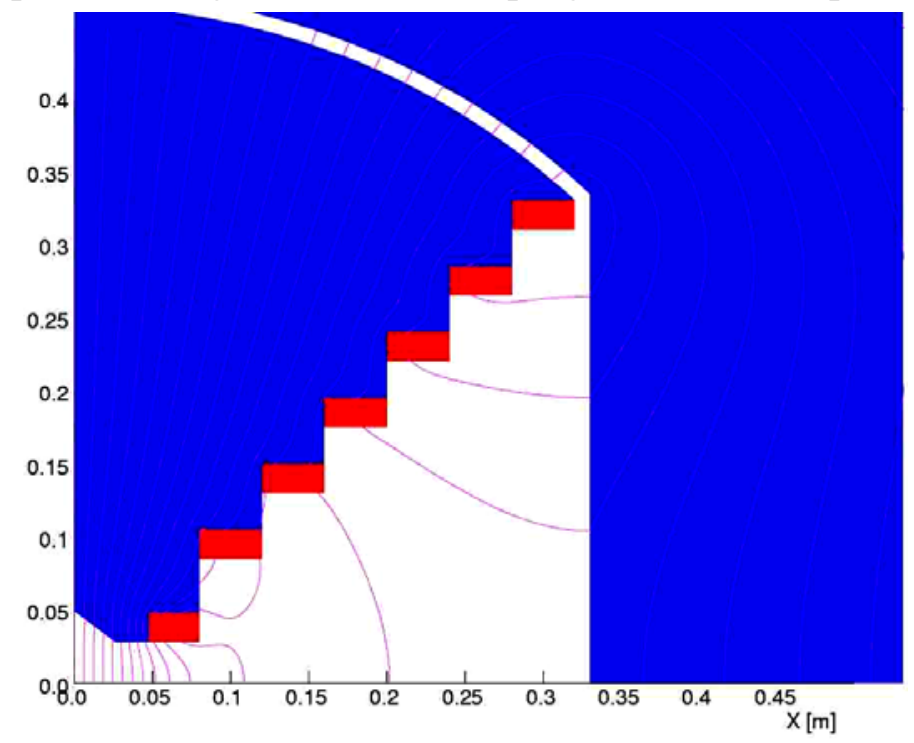

Figure 33. Levitated-pole dipole, 8.3 Tesla in a $56 \mathrm{~mm}$ clear slot aperture.

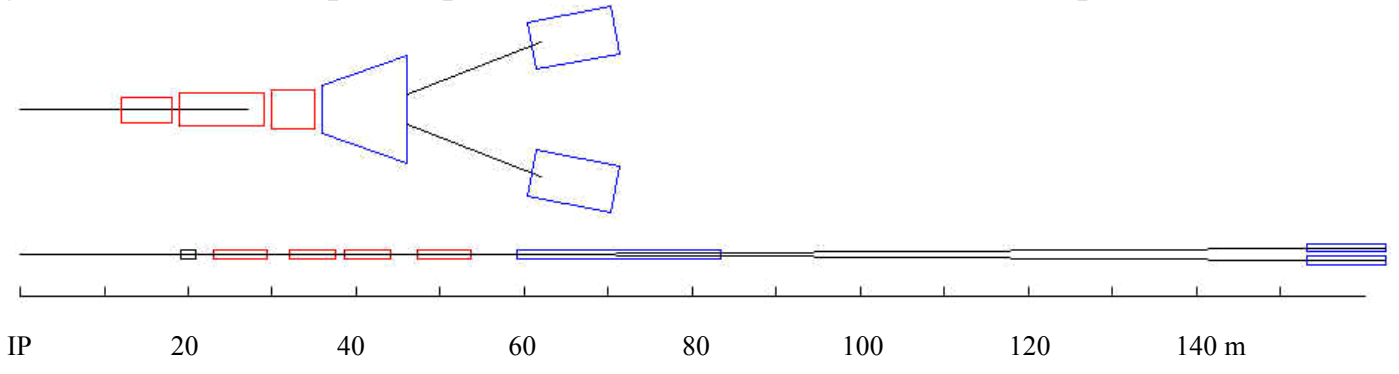

Figure 34. Comparison of compact IR (top) and baseline IR (bottom) for LHC. 


\section{Hybrid dipoles for an LHC Tripler}

The 2003 Erice Workshop inspired us to examine how to extend the benefits of our stress management structure and flux plate magnetics to magnet designs with field strength even beyond the $\sim 16 \mathrm{~T}$ that can be attained with $\mathrm{Nb}_{3} \mathrm{Sn}$. That requires the use of $\mathrm{Bi}-2212$ windings, with an entirely new set of challenges. We have developed a design for a hybrid dipole that would contain such Bi-2212 inner windings and $\mathrm{Nb}_{3} \mathrm{Sn}$ outer windings ${ }^{5}$. We authored a conceptual design for a 24 Tesla dual dipole using that approach, with the same size and aperture as LHC, which could provide a way to triple the energy of LHC in a future upgrade in the same tunnel.

We also devised a solution to one obvious difficulty that such an upgrade would engender the synchrotron radiation power would increase like the $4^{\text {th }}$ power of field strength, and the cryogenic heat load from synchrotron light is already a significant issue for LHC. We solved that ancillary problem with photon stops, integrated into the dipole itself, on which the synchrotron light is absorbed at a much higher reservoir temperature $(160 \mathrm{~K})$ so that cooling the heat is not a limiting load on the cryogenic system.

The LHC Tripler proposal has sparked considerable interest in the international community. We are submitting a separate new proposal to the AARD program to support the first stages of development of Bi-2212 winding technology. There is no margin of resources in our base program to support that development. The base program will proceed with developing TAMU4 and optimizing and validating all of its design features. The hybrid dipole uses exactly the same magnet design and structures as TAMU4. The parallel thrusts of our base program for TAMU4 and the new proposal for development of Bi-2212 winding technology form the critical path to develop the possibility of an LHC Tripler.

The development of the Bi-2212 winding technology will require a major development effort. A collaboration is being developed among Texas A\&M, LBNL, Los Alamos, Showa Wire, and the University of Twente. Funding will be proposed to NSF and DOE.
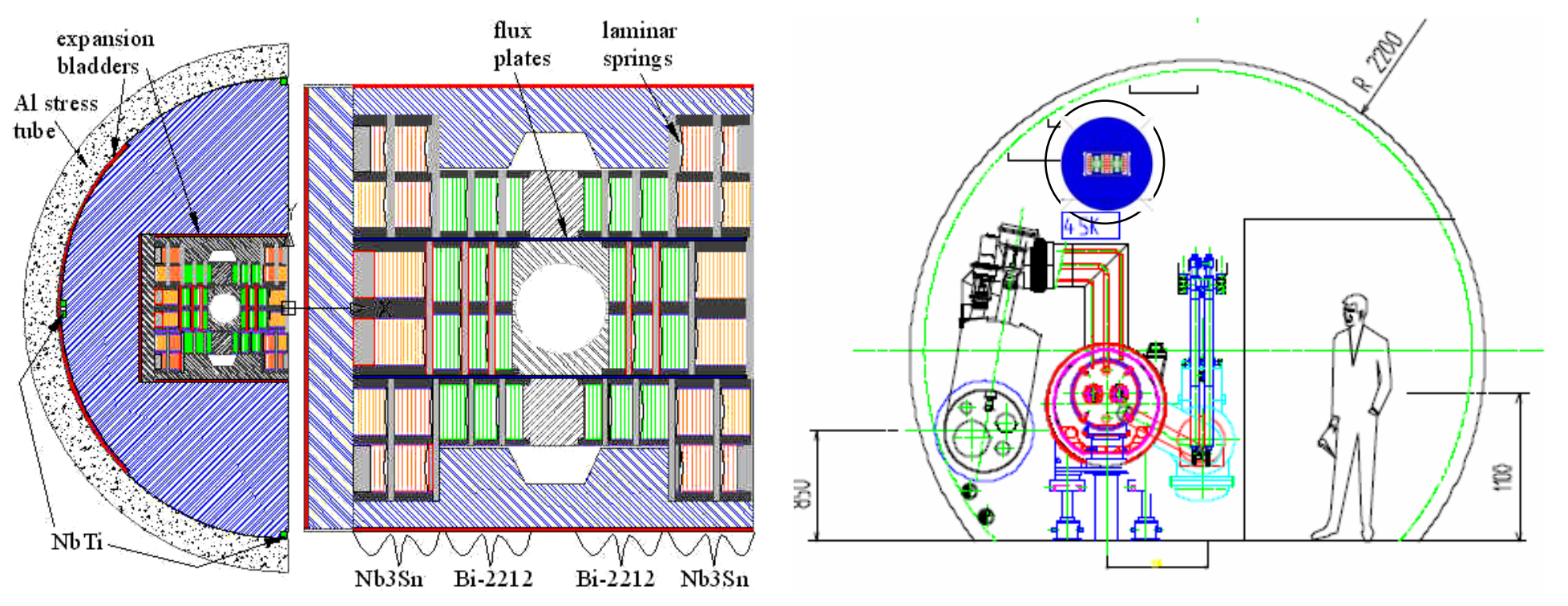

Figure 35. 24 Tesla hybrid dipole for the LHC Tripler. 


\section{Key Personnel}

Peter McIntyre holds the Mitchell/Heep Chair in Experimental Physics at Texas A\&M University, and has led the development of the block-coil design to date. In the past he has authored the concepts of proton-antiproton colliding beams, invented depressed-collector techniques for electron cooling, co-authored the superferric design of the SSC, and developed cathode technology for high-power microwave tubes. Prof. McIntyre is now also contributing to an industry effort towards new manufacturing technology for high-performance internal-tin $\mathrm{Nb}_{3} \mathrm{Sn}$ superconducting wire.

Al McInturff is a senior accelerator physicist, with 40 years of experience in superconductor development and the design, fabrication, and testing of superconducting magnets. Dr. McInturff has contributed to every facet of superconducting technology, strand development, PIT process in $\mathrm{Nb}_{3} \mathrm{Sn}$, development of the successful Tevatron magnets, development and testing of the SSC dipoles, and most recently the pattern of successful developments of $\mathrm{Nb}_{3} \mathrm{Sn}$ dipoles at LBNL.

Akhdiyor Sattarov was trained as a nuclear theorist, and has been working for the past 5 years in the calculational physics of superconducting magnets and particle accelerators. He performs finite element calculations of the magnetic fields, the mechanical stress, and the quench propagation in the magnets, and also beam optics and dynamics in accelerators. He has developed techniques by which to do constrained quadratic optimization of the dipole designs.

Tim Elliott is a senior technician, with 20 years experience in a wide range of technologies. He contributes to the mechanical designs of both magnets and fixtures, electrical controls and DAQ. He operates our high vacuum systems and hydraulic systems. He is also our EDM machinist, and has developed the CAD/CAM links by which we are able to make parts efficiently.

Bill Henchel is a senior technician, with 22 years experience in cryogenics, electrical, and mechanical technology. He worked at Fermilab, in the cryogenics and CDF groups, before joining our group. Bill is the person in charge of our coil winding and associated fixtures. He has developed the designs for many of the detail subassemblies that are critical to stress management and to the integration of the magnet into a consistent whole.

Nick Diaczenko is a senior machinist and technician, with extensive experience in the fabrication of precision equipment of all kinds. Nick developed our laminar spring, machined many of the parts in our magnet assembly, and developed the demanding weld techniques for the hoop plates, the body welds, and the high-pressure bladders.

Ray Blackburn is our CAD designer. He has designed all of the piece parts and assemblies for the dipoles described above. He took the lead in developing our systems for cable cleaning and spray application of palmitic acid. He is building the support coffin that will hold the coil assembly during reaction bake.

Andrew Jaisle is a senior technician, who participated in the magnet R\&D team at Brookhaven, then ran the model magnet fabrication facility at SSC. Andrew worked with us for two years earlier, then returned to the Dallas area for personal reasons. He has elected to return to the group, and is taking leadership straightaway in the completion of the furnace lift fixture and the modifications of the impregnation system.

In addition we have four graduate students - two in their third year (Joong Byeon and Patrick Noyes) and two in their first year (Eiichi Hirose and Amanda Woodward). Four undergraduate students (Michael Hatridge and Ryan Schmidt) participate in the research. All of these students are academically strong and well motivated. 


\section{References}

${ }^{1}$ R. Blackburn et al., "Construction and testing of block-coil high-field dipoles for future hadron colliders", Proc. Appl. Superconductivity Conf., Houston, TX, August 4-9, 2002.

${ }^{2}$ P. McIntyre, R. Scanlan, and W. Shen, "Ultra-high field magnets for future hadron colliders", Proc. Appl. Superconductivity Conf., IEEE Trans. on Appl. Superconductivity 5, \#2, June 1995, pp. 1099-1102.

${ }^{3}$ N. Diaczenko et al., "Stress management in high-field dipoles", Proc. 1997 Particle Accelerator Conf, Vancouver, May 12-16, p.3443 (1997).

${ }^{4}$ D. Finley, D. Edwards, R. Hanft, D. Johnson, A. McInturff, and J. Strait, "Time dependent chromaticity changes in the Tevatron", Proc. Particle Accel. Conf. (PAC87), Washington, DC, IEEE Cat\#: 87CH2387-9, pp. 151 - 153.

S. Sanfilippo, "Persistent and coupling current effects in the LHC superconducting dipoles", Proc. Appl. Superconductivity Conf., Houston, TX, August 4-9, 2002.

${ }^{5}$ P. McIntyre and A. Sattarov, "Tripling the LHC: the path from technology to discovery, Proc. DARK2004 Conference on dark matter in the universe", Springer (2004).

P. McIntyre and A. Sattarov, "On the feasibility of a tripler upgrade for LH”, Proc. Particle Accel. Conf., Knoxville, TE, May 16-20, 2005.

${ }^{6}$ C. Battle et al., "Optimization of block-coil dipoles for hadron colliders", Proc. Particle Accelerator Conf., New York, NY, March 30-April 1, 1999.

${ }^{7}$ R. Hafalia, et al., “A new support structure for high field magnets”, Proc. of MT-17, Geneva, Suisse, IEEE Trans. on Appl. Superconductivity 1, pp. 47 - 50.

${ }^{8}$ S. Caspi, "The use of pressurized bladders for stress control of superconducting magnets", Proc. Appl. Superconductivity Conf., Virginia Beach, VA, September 17-22, 2000, LBNL-45180, SC-MAG-706.

${ }^{9}$ M. Adams et al., “Accelerator-driven thorium cycle: new technology makes it feasible”, Proc. Int'l Congress on Advanced Nuclear Power Plants, Hollywood, FL June 9-13, 2002.

${ }^{10}$ Magnetization data measured by R. Goldfarb, NIST.

${ }^{11}$ R. Blackburn et al., "12 Tesla hybrid block-coil dipole for future hadron colliders", Proc. Applied Superconductivity Conf., Virginia Beach, VA Sept. 17-22, 2000.

${ }^{12}$ D. Dietderich, J. Litty, and R. Scanlan, "Dimensional changes of $\mathrm{Nb}_{3} \mathrm{Sn}, \mathrm{Nb}_{3} \mathrm{Al}$, and $\mathrm{Bi}_{2} \mathrm{Sr}_{2} \mathrm{CaCu}_{2} \mathrm{O}_{8}$ conductors during heat treatment and their implications for coil design", Advances in Cryogenic Engineering (Materials) 44B, Plenum Press, NYC, NY, pp. 1013-1020 (1998).

${ }^{13}$ A.D. McInturff, "Quench protection for high field magnets", Workshop on VLHC Magnet Technologies, Fermilab, May 24-26, 2000.

14 P. McIntyre and A. Sattarov, 'Killing the electron cloud effect in the LHC arcs', Proc. Particle Accel. Conf., Knoxville, TE, May 16-20, 2005.

${ }^{15}$ A. Sattarov, J-P. Koutchouk, and P. McIntyre, 'Towards an optimization of the LHC intersection region using new magnet technology', ibid.

${ }^{16}$ N. Diaczenko et al., 'Stress management of HTS conductor', Proc. Appl. Superconductivity Conf., Palm Desert, CA, September 25-30, 1998.

G. Liang et al., 'Strain-tolerant cable using Bi-2212 superconductor', Proc. Int'l. Conf. on Magnet Technology, Jacksonville, FL, Sept. 26-30, 1999. 